\title{
From Thought to Practice: Appropriation and Endogenous Market Structure with Imperfect Intellectual Property Rights ${ }^{1}$
}

\author{
Mariagiovanna Baccara ${ }^{2}$ and Ronny Razin ${ }^{3}$
}

April 2002

\footnotetext{
${ }^{1}$ We are grateful to Patrick Bolton, Gene Grossman, Alessandro Lizzeri and Wolfgang Pesendorfer for advising us on this project. We also thank Jean-Pierre Benoit, Kfir Eliaz, Faruk Gul, Hugo Hopenhayn, Marciano Siniscalchi, Jan Zabojnik and the participants of seminars at Princeton University, New York University, Marshall School of Business (USC), Rutgers University, Carnegie Mellon University, Washington University, University of Southern California, Columbia Business School, Stern School of Business (NYU), University of Toronto, Stanford GSB, University of Rochester, Bocconi University, IGIER and the V SAET Conference in Ischia.

${ }^{2}$ Department of Economics, Princeton University, mariagb@princeton.edu

${ }^{3}$ Department of Economics, New York University, ronny.razin@nyu.edu
} 


\begin{abstract}
We address the problem faced by innovators who have an idea for a marketable product but must hire employees to bring the product to the market. Information leakage implies that newly hired employees become informed of the idea and may attempt to bring the product to the market themselves. We develop a bargaining model to analyze this situation. In this model, employees' rents endogenously reflect the bargaining power vis-a-vis the firm which is due to the knowledge of the information. The model has a unique symmetric equilibrium in which the innovator appropriates a sizable share of the surplus despite the absence of property rights for ideas. We show that this share stays bounded away from zero even as the number of agents required in the development grows to infinity. We also derive the conditions under which monopoly or competition arise on the product market. We find that when the degree of potential competition on the product market is high enough, a monopoly is generated by hiring all potential competitors within the same firm. Finally, the link between intellectual property rights enforcement and industry performance is explored, and normative implications are derived.
\end{abstract}




\section{Introduction}

In this paper we analyze the process of innovation when Intellectual Property Rights (IPR) are not perfectly enforceable. We focus on two main issues: the innovators' appropriation rates and the market structure that may endogenously be determined by information leakage concerns.

To better understand these two issues, consider the following analogous story. A person finds a map illustrating the location of a treasure on an island. In order to reach the island, this person needs the help of others. In the process of trying to recruit help, information on the whereabouts of the treasure may leak. At this point, nothing prevents the new recruits from leaving the owner of the map and trying to find the treasure with someone else.

A first set of questions arises because the information laid down on the map is not protected. How is the map owner's position affected by the risk of information leakage? What share of the treasure would he have to offer to his companions? Will he have enough incentives to try and look for the treasure?

An additional set of issues arises after the treasure has been found. Assume that the first team to reach the treasure is not protected against others trying to steal it. The holders of the treasure are therefore always at risk of being robbed, as long as other people know about its existence.

More specifically, consider a group of people who initially know about the map. Will they reach the treasure all together as one team? If they do, they will not face the risk of robbery after they find it. On the other hand, the treasure will have to be split among many people. Alternatively, it could be that some in the group may have an incentive to form a smaller team. Small teams involve less people to share the pie among. However, as these teams may end up robbing one another, the pie each of them will share is smaller. Do we expect to see one team enjoying the treasure or numerous teams fighting for it at the end of the day?

As for innovation, it starts with an idea. The idea evolves into a new product, and then the product enters the market. Developing an idea from the initial concept to a marketable commodity is a process that exposes inventors to different kinds of risks.

Information sharing is often necessary to the development process. When information has been shared and employees defect, an innovator may loose control of his idea. Employees' defection can hurt an innovator in two fundamental ways. First, defection may result in a race to hit the market first (reaching the treasure). This could mean the loss of any rents innovators could secure by an incumbency advantage. Second, defection may result in future competition in the market for the 
final product (fighting over the treasure). Even if an incumbent advantage is assured, innovators' profits may decrease due to the presence of new competitors.

Legal systems may provide means of protecting innovators' ideas by enforcing IPR. ${ }^{1}$ In practice, the enforcement of these rights is often problematic. ${ }^{2}$ In fact, the intrinsic nature of ideas makes it difficult to define property rights and to prove their violations. Moreover, it has been suggested that the degree of enforcement of property rights, which changes across states and industrial regions, may influence the configuration of local industries and their fortunes. ${ }^{3}$ This raises questions about the relation between the enforcement of IPR and the performance of industries.

We analyze a model of the formation of new firms in the absence of perfect IPR. In the model agents make offers to other agents to start a collaboration on a new business idea. The crucial feature of the model is the presence of information leakage. We assume that the development of an idea requires collaboration and that collaboration entails information sharing.

This environment suggests a natural protocol for a bargaining game. Suppose an innovator has an idea that needs to be developed with at least one other agent. At the beginning of the bargaining game, the innovator can make an offer to any subset of a pool of agents. All agents who receive the offer learn the idea, i.e. become "informed". If the offer is accepted by everybody, a firm forms and the development takes place. If the offer is rejected by someone, a new negotiation ensues. As the set of informed agents is enlarged, the race to carry out the development is more intense. We model the intensity of the race by assuming that the probability any agent makes the next offer is inversely related to the number of informed agents.

The continuation of the game after one firm has formed depends on the legal regime under scrutiny. We consider two legal regimes. First, in a Partial Protection regime, ideas are not protected during the development stage, but patents are granted when the development is completed.

\footnotetext{
${ }^{1}$ The enforcement of no-compete agreements and, to some extent, trade secret law protect inventors from information leakage during the development stage. The patent system aims to guarantee inventors a monopoly on the product market.

${ }^{2}$ Areeda and Kaplow (1998) argue that it is often the case that even if patents are granted, it is considered unlikely they will stand in court if ever litigated. Besen and Raskind (1991) and Fisk (2001) discuss the limitations of the enforcement of trade secrets law and no-compete agreements.

${ }^{3}$ See Hyde (2000) and Gilson (2000) for the comparison between the Silicon Valley and the Route 128 (Mass.) industrial regions. Both these studies stress how, in contrast with common wisdom, the weak enforcement of nocompete clauses granted by California law may have contributed to the success of Silicon Valley. His argument rests mainly on the assumption that weak IPR imply information diffusion, which in turn stimulates incremental research and hence innovation.
} 
In this case, the game ends when an offer is accepted and the first firm forms. ${ }^{4}$

Second, we analyze a No Protection regime. In this regime, patents cannot be granted at the end of the development stage. If this is the case, if the first firm to form does not employ all the informed agents, the game continues. The informed agents that are still unemployed can apply the idea to an identical or similar product and generate competition in the final product market.

We measure the appropriation rates of innovators operating under the different legal regimes. In the Partial Protection regime, we find that there is a unique equilibrium in which the innovator always receives a surprisingly high share of the profits. ${ }^{5}$ In particular, if two agents are necessary to develop the product, as the bargaining frictions disappear the share of the innovator goes to $1-e^{-1}$, i.e. $63 \%$ of the profits. If the number of people necessary to develop the product goes to infinity, the appropriation rate of the innovator goes to $e^{-1}$, i.e. $34 \%$ of the profits. This means that the information leakage problem does not dissipate an innovator's payoff even when the idea requires many employees and forces him to face the potential defection of all of them.

The innovator's success is due both to a first-mover advantage (that disappears as frictions become negligible) and to a second, novel effect that is robust to the disappearance of bargaining frictions. We refer to this effect as the "information diffusion advantage." 6

The intuition behind the information diffusion advantage is the following. Suppose that there are four agents in the game. Agents 1 and 2 are informed about the idea, and agents 3 and 4 are not. Suppose that agent 1 is chosen as next proposer. He has to decide whether to form a firm with agent 2 , who is already informed, or to diffuse the information further by hiring a new agent, say agent 3 . The more agents that are informed about the idea, the higher the competition in the race to make the next offer. This suggests that agent 3 maybe cheaper to hire than agent 2 , so that diffusing the information is preferable. On the other hand, if the continuation values of the agents decrease as the number of informed agents increases, by making an offer to an uniformed agent, agent 1 lowers the wage agent 3 will have to pay his employee, i.e. agent 4, upon rejection, in the event agent 3 will become the next proposer. This suggests that a proposer may want to

\footnotetext{
${ }^{4}$ The analysis of this legal scenario in the presence of information leakage captures a novel aspect of patent races. Any team of agents working to develop a patentable product are subject to the risk of competition arising from within. Dasgupta and Stiglitz (1980), Lee and Wilde (1980) and Loury (1979) have analyzed patent races, but these papers do not analyze the infomational aspects of the development stage.

${ }^{5}$ In our analysis, we focus our attention on the set of the Symmetric Subgame Perfect Equilibria.

${ }^{6}$ For a competitive treatment of information diffusion, see Chari and Hopenhayn (1991) and Jovanovic and McDonald (1994).
} 
keep the number of informed agents low, and hire an informed agent rather than an uninformed one. We show that the first effect is stronger. This implies that information gets diffused to more and more new agents off the equilibrium path. The net effect, or the information diffusion advantage, works in favor of the innovator as it decreases the continuation value of her employee.

Let us now turn to the No Protection regime. In the total absence of IPR, patents are not available to guarantee a monopoly at the end of the development process. An agent who is about to make an offer has to consider the effect of his offer on the final product market structure. Besides the potential defection of the agents he hires, a proposer has to evaluate the threat of future market competition. Thus, he faces an additional trade-off. In particular, he can either preempt the potential competition on the final product market by hiring all the agents that could potentiality compete with him (i.e. all the informed agents), or he can form a firm in the least expensive way, and face competition on the final product market.

We show that even in the absence of any kind of IPR two equilibrium effects enable innovators to appropriate significant shares of the profits. First, we show that for a range of parameters the innovator still enjoys the information diffusion advantage. Second, higher rents for the innovator can be sustained by a new effect that we term the Threat of Competition Advantage. We show that the innovator is sometimes able to credibly threaten his employees of competition arising upon rejection of his offer. As competition dissipates rents, this tends to lower the continuation value of potential employees. Thus, this threat enables the innovator to appropriate all of the gap between monopoly rents and the total rents under market competition. This implies that the fiercer is the potential market competition, the stronger is the position of the innovator.

We show that there is a limit to the ability of the innovator to use the threat of competition. In particular, when the degree of potential market competition is very high, the threat of future competition is not credible any longer. Upon rejection of an offer, agents always have incentives to avoid competition and they tend to make offers that generate monopoly. This keeps the innovators' payoffs at the minimum as both the information diffusion and the threat of competition advantages cease to hold.

Our analysis of the No Protection regime offers predictions on information diffusion and market structure outcomes when several agents are initially informed about the idea. We show that, even in absence of IPR, it may very well be that monopoly is the market outcome. This happens if the degree of potential market competition is high, and in this case, all information remains 
concentrated inside the boundaries of a single firm. ${ }^{7}$ On the other hand, if the degree of potential competition on the final product market is low, competition is the equilibrium market outcome.

Both economists and legal scholars are involved in an ongoing debate about the optimal definition, extent, and enforcement of IPR. ${ }^{8}$ This debate aims to identify costs and benefits of such protection in terms of economic efficiency. From our results, we derive normative implications. ${ }^{9}$ First, our paper contributes to the literature on the optimal definition of IPR suggesting that the impact of IPR on incentives to innovate has been generally overestimated. In particular, our results show that, in the absence of perfect IPR, equilibrium effects protect inventors' payoffs from dissipation.

Second, our results also have important normative implications for the relation between the enforcement of property rights and industry performance. In our model, employees are paid according to the information they hold and not according to their productivity. In the presence of many agents competing for the same job, one would expect that employee rents would be low. In our model, in contrast, information leakage creates a wedge between informed and uninformed agents, giving the informed ones bargaining power vis-a-vis the employer. This entails strictly positive rents for employees.

In an extension of the model discussed in Section 5.2, when the development process requires a two-sided, non-contractable investment by employer and employee, perfect property rights protection may result in investment levels that are inefficient. Our model provides an endogenous determination of the division of rents within the firm. We provide an example in which the presence of information leakage entails more efficient effort levels within firms compared with the case of perfect protection of IPR.

The first ones to approach the informational concerns of inventors in the absence of IPR have been Anton and Yao (1994). Anton and Yao present a model in which a financially weak inventor faces two manufacturers that can market his product. In the negotiation with the first manufacturer, he can increase his bargaining power by threatening to reveal his idea to a competitor. The main difference between their approach and ours is their assumption that, once they learn the idea, manufacturers do not face problems of information leakage. Our approach rests on the observation that in the absence of property rights, a manufacturer who employs some workers

\footnotetext{
${ }^{7}$ This is in line with studies suggesting that many firms rely on secrecy more than on patent protection to protect their R\&D. See Levin, Klevorick, Nelson and Winter (1987).

${ }^{8}$ Among many, see Arrow (1962), Hughes (1988), Ordover (1991), Scotchmer (1991).

${ }^{9}$ We further discuss normative implications in Section 5 .
} 
may eventually face the same information leakage problem that the innovator faced in the first place. This difference in the approach suggests an alternative bargaining model and significant differences in the results, that we discuss in detail in Section 3.

Our model also relates to the notion that the employees of a firm could be tempted to appropriate the source of the rent of the firm. Rajan and Zingales (2001) carry out an analysis of the optimal design of a hierarchy to prevent employees from doing so. The difference in the focus of the analysis is reflected in the modelling assumptions: in their model, employees bargain on their wage after deciding whether to stay in the firm or to defect. In our model the defection decision can occur at any moment between the bargaining and the completion of the development process, so it affects the outcome of the bargaining. ${ }^{10}$

The paper is structured as follows. In Section 2, we present the model and a benchmark in which IPR are perfectly enforced. In Section 3, we analyze the Partial Protection regime. In Section 4 we discuss the No Protection regime. In Section 5, we discuss the main assumptions and the normative implications of the model.

\section{The model}

The strategic situation outlined in the introduction suggest a natural protocol for a bargaining model. Three assumptions underlie this protocol. ${ }^{11}$ First, we assume that no agent can develop an idea into a product on his own. Second, we assume that the act of recruiting entails sharing information about the idea. Finally, our third assumption relates to the asymmetry between informed and uninformed agents. The only element differentiating otherwise homogeneous agents is the knowledge of the idea. We capture this asymmetry by assuming that offers can be made only by informed agents. ${ }^{12}$

\footnotetext{
${ }^{10}$ For other papers that relate the possibility of employees' defection to the distribution of wages within the firm, see Stole and Zweibel (1994), Wolinsky (2000) and Zabojnik (2000). In particular, Zabojnik (2000) explores how hierarchical firms pay employees efficiency wages in accordance with the potential threat of their leaving the firm with relevant information. The common element between Zabojnik's and our paper is the idea that wages are not fully explained by the productivity of the employees but rather they incorporate the relevance of the information employees hold. However, while Zabojnik models the threat as an external technology, we endogenize the process that makes this information a threat for the employer by explicitly modeling the continuation game.

${ }^{11}$ The assumptions of this model are discussed further in Section 5.

${ }^{12}$ This assumption is motivated by the uninformed agents being unaware of the existence of the idea or of its potential profitability. They become aware of it only when approached by an informed agent for the first time.
} 
We now present the model in detail. Let us consider a finite set of agents $N$, with $\operatorname{card}(N) \equiv$ $n>2$, among which there is a set of innovators $K^{0}=\left\{1,2, . ., k^{0}\right\}$ that have an idea for a business venture. All the agents in $N \backslash K^{0}$ are initially unaware of the business idea. If developed, this idea can be implemented into one or more marketable products. The process of developing the idea requires the work of several agents. First, we assume, for simplicity, that the work of exactly two agents is required to turn the idea into each marketable product. ${ }^{13}$

Knowledge of the idea is necessary for any group of agents to develop the idea into products. Thus, if all the informed agents are in one firm, this firm enjoys a monopoly in the product market. Any knowledgeable agent who is not part of any existing firm can always try and form her own firm and develop the same or a similar product. This new firm will compete to some degree with the first firm on the final product market. We assume that the market can accommodate only two firms. We normalize the present value of all the profits earned by the first firm if the second firm never enters the market to be equal to 1 . Let $\pi_{2}$ be the present value of all the profits earned by the second firm in competition. Then, notice that the present value of all the profits earned by the first firm is increasing in the delay with which the second firm enters the market. We let $\pi_{1} \leq 1$ be the present value of all the profits of the first firm if a second firm forms after one period. ${ }^{14}$ Since the incumbent firm markets neither the second product nor both products, we assume $\pi_{1} \geq \pi_{2}$ and $\pi_{1}+\pi_{2} \leq 1$. $^{15}$

The structure of the game builds recursively on two types of negotiation subgames. What distinguishes the two types of subgames is whether one firm has already formed or it has not.

Suppose we are at some history along the game at which a firm has not yet formed and the set of the informed agents, i.e. the agents who know the idea, is $K^{\prime} \supseteq K^{0}$. We are now ready to introduce the first negotiation subgame. We assume that nature chooses with equal probability among the informed agents in $K^{\prime}$ the next agent to make an offer. The chosen agent, say agent

\footnotetext{
${ }^{13}$ In Section 3.1, this assumption is relaxed.

${ }^{14}$ Let product $\pi_{1}^{m}$ and $\pi_{2}^{m}$ be the monopolistic profit earned in one period by a firm producing the first and the second product, respectively. Let also $\pi_{1}^{c}<\pi_{1}^{m}$ and $\pi_{2}^{c}<\pi_{2}^{m}$ be the profits earned in one period by a firm producing the first and the second product, respectively, when both products are present on the market. Let the first product be the most profitable one, i.e. $\pi_{1}^{m} \geq \pi_{2}^{m}$ and $\pi_{1}^{c} \geq \pi_{2}^{c}$. Observe that if a second firm enters the market $x$ periods after the first one, the first firm earns $\pi_{1}^{m}$ for $x$ periods, and then it starts earning $\pi_{1}^{c}$ from then on. The payoff of the first firm is $\pi_{1}(d)=\pi_{1}^{m} \frac{1-\delta^{d}}{1-\delta}+\pi_{1}^{c} \frac{\delta^{d}}{1-\delta}$. However, since a second firm never forms after more than one period after the first one, nothing changes in our analysis if we just assume the payoff of the first firm always to be equal to $\pi_{1} \equiv \pi_{1}(1)$.

${ }^{15}$ See Section 5.1 for a discussion of the implications of relaxing the hypothesis $\pi_{1}+\pi_{2} \leq 1$.
} 
$i \in K^{\prime}$, can propose a division of the surplus to a set of agents $C^{\prime} \subset N \backslash\{i\}$. The division of the surplus, $\alpha$, is a vector $\alpha \in \Delta^{\operatorname{card}(C)}$, where $\Delta^{\operatorname{card}\left(C^{\prime}\right)}$. is the simplex in $R^{\operatorname{card}(C)}$ An offer is fully represented by the pair $\left(C^{\prime}, \alpha\right)$. The agents in $C^{\prime}$ have to decide simultaneously whether to reject or to accept the offer. The crucial assumption in this model is that all of the agents who receive an offer become informed, and the set of the informed agents becomes $C^{\prime} \cup K^{\prime}$. If at least one agent in $C^{\prime}$ rejects the offer, they enter a negotiation subgame in which no firm has formed. If all accept, then the first firm is formed, and two resulting cases are possible.

If $C^{\prime} \supseteq K^{\prime} \backslash\{i\}$, i.e. all the other informed agents are included in the offer, then the game ends; the firm implements the idea and enjoys a monopoly status. Any agent $j \in C^{\prime}$ receives $\alpha_{j}$, agent $i$ receives $\left(1-\sum_{j \in C^{\prime}} \alpha_{j}\right)$, and agents in $N \backslash\left(C^{\prime} \cup\{i\}\right)$ receive zero. We refer to an offer such that $C^{\prime} \supseteq K^{\prime} \backslash\{i\}$ as a "grand coalition" offer. If $C^{\prime} \nsupseteq K^{\prime} \backslash\{i\}$, not all the informed agents become part of the first firm. The informed agents that are not part of the first firm can keep on negotiating and form a second firm. We therefore enter a second type of negotiation subgame in which one firm has formed and for which the set of informed agents left in the game is $K^{\prime} \backslash\left(C^{\prime} \cup\{i\}\right)$. In any terminal node following this history agent $i$ receives $\left(1-\sum_{j \in C^{\prime}} \alpha_{j}\right) \pi_{1}$ and any agent $j \in C^{\prime}$ receives $\alpha_{j} \pi_{1}$. We refer to an offer such that $C^{\prime} \nsupseteq K^{\prime} \backslash\{i\}$ as a "cost minimizing" offer.

Let us now introduce the second type of negotiation subgame. Such subgames ensue after some agent $i$ has already formed a firm making a successful offer to the set of agents $C^{\prime}$. Let $K^{\prime \prime}$ be the set of informed agents left in the game. With equal probability, an agent $h$ is chosen from $K^{\prime \prime}$ to propose a division of the surplus to a set of agents $C^{\prime \prime} \subset N \backslash\left(C^{\prime} \cup\{i\} \cup\{h\}\right)$. Let $\beta \in \Delta^{\operatorname{card}\left(C^{\prime}\right)}$ be the proposed division. If everybody accepts the offer, the game ends, agent $h$ receives $\left(1-\sum_{j \in C^{\prime \prime}} \beta_{j}\right) \pi_{2}$, and any agent $j \in C^{\prime \prime}$ receives $\beta_{j} \pi_{2}$. All the agents in $N \backslash\left(C^{\prime} \cup C^{\prime \prime} \cup\{i\} \cup\{h\}\right)$ receive zero. If someone in $C^{\prime \prime}$ rejects offer $\beta$, then the we enter a negotiation subgame in which one firm has formed and for which the set of informed agents is $K^{\prime \prime} \cup C^{\prime \prime}$.

Note that we use unanimity as the rule that governs the formation of a firm, so that the offers are conditional upon the acceptance of all the agents involved. This implies that agents cannot make an offer that is binding on his side as soon as at least one agent accepts it ("unconditional offer"). ${ }^{16}$

The game begins with a negotiation subgame for which the set of informed agents is $K^{0}$. We assume that there are frictions in bargaining due to impatience. These frictions are represented

\footnotetext{
${ }^{16}$ In Section 5, this assumption is discussed in detail.
} 
by a common discount factor $\delta \in(0,1)$. Every time we enter a negotiation subgame, payoffs in that subgame are discounted by $\delta$. If no agreement is reached, we assume payoffs are zero. All the agents have reservation value normalized to zero and are risk-neutral.

Before we specify the notion of equilibrium we adopt, let us introduce the set of possible histories of this game, $H$. The set $H$ can be decomposed into the subsets $H_{O}, H_{R}, H_{N}$ and $H_{T}$. The set $H_{O}$ includes all the histories at which an agent is called to make an offer, and we denote by $h_{i}$ a generic history in $H_{O}$ at which agent $i$ is called to make an offer. The set $H_{R}$ includes all the histories at which agents are simultaneously called to reply to an offer, the set $H_{N}$ includes all the histories at which nature chooses the next proposer, and the set $H_{T}$ include all the terminal histories. Every history in $H_{O}$ is followed by a history in $H_{R}$, and every history in $H_{R}$ is followed either by a history in $H_{T}$ or by a history in $H_{N}$. Every history in $H_{N}$ is followed by a history in $H_{O}$. Let $K(h)$ be the set of informed agents in the game at history $h \in H$, and let $k(h) \equiv \operatorname{card}(K(h))$.

For any player $i \in N$, a strategy $s_{i}$ is defined for all histories in $H$ at which agent $i$ takes an action, specifically for all histories in $H_{O}$ at which he is called to make an offer and all histories in $H_{R}$ at which he is called to reply.

To analyze this model, we look at Symmetric Subgame Perfect Equilibria (SSPE). ${ }^{17}$ Among the SSPE, we look at those in which agents do not use weakly dominated actions when responding to offers. ${ }^{18}$

In the analysis of the model, we compute the continuation values of the players at histories $h \in H_{N}$, i.e., when nature is about to choose the next proposer and the payoffs are about to be discounted. We denote the continuation value of agent $i$ at a given history $h$ as $v^{i}(h)$.

A property of the SSPE is that for any $h \in H_{N}$, all the informed agents have the same continuation value, or $v^{i}(h)=v^{j}(h)=v(h)$ for all $i, j \in K(h) .{ }^{19}$

\footnotetext{
${ }^{17}$ For the formal definition of SSPE we refer to the Appendix. In Section 5, we discuss the implications of restricting attention to SSPE.

${ }^{18}$ This assumption is important only in Subsection 3.1 and Section 4, where situations in which proposers are forced to make offers to more than one agent become relavant. We want to rule out equilibria that are sustained by the mere fact that agents are not pivotal. For example, one can sustain equilibria in which offers to more than one agent are never accepted by any agent. These strategies could be chosen in equilibrium as, by our unanimity assumption, no agent is pivotal in the acceptance or rejection of such offer. By assuming away weakly dominated actions, we guarantee that an agent who desires the offer to be accepted, votes in its favor.

${ }^{19}$ In the remaining of the paper, we often analyze equilibria in which the continuation values of the informed agents are the same at any history characterized by the same number of informed agents (i.e. $v(h)=v\left(h^{\prime}\right)$ for any $h, h^{\prime} \in H_{N}$ such that $\left.k(h)=k\left(h^{\prime}\right)\right)$. If this is the case, we use the notation $v_{k(h)} \equiv v(h)=v\left(h^{\prime}\right)$.
} 


\subsection{The Perfect Protection Benchmark}

Before presenting the benchmark of our model in the case in which IPR are perfectly defined, we want to make a distinction between two different types of protection and their respective enforcement. As we already mentioned in Section 1, the presence of information leakage exposes innovators to two fundamental risks. When the inventor initially contacts people to start the development of the idea, he faces the possibility that employees will defect and start their own development team. A perfect protection against this kind of risk should be able to guarantee that in the case in which the project is not developed by the original inventor, the identity of the inventor can be perfectly observed, and he gets the restitution of the full value of his own idea. ${ }^{20}$

When a firm introduces a product on the market, it faces the possibility that other firms may compete with the same or a similar product. Perfect protection against market competition guarantees that when competition appears, monopolistic profits can be computed and awarded to the first firm. ${ }^{21}$

As a benchmark of our model, we analyze what happens if both means of IPR are perfectly enforceable. We therefore assume the following two assumptions. First, the completion of the development of an idea guarantees a monopoly on the final market. In our model, this amounts to assuming that $\pi_{1}=1$ and $\pi_{2}=0$. Second, innovators have the right to collect the profits earned by their ideas. This implies that when there are $k_{0}$ initial innovators, every innovator, at any terminal history in which he is not included in the firm that develops the idea, can obtain the restitution of $\frac{1}{k_{0}} \cdot{ }^{2223}$

We claim that when IPR on ideas are perfectly protected, in all SSPE innovators appropriate the full value of their ideas. ${ }^{24}$ Consider the case of a single innovator. Note first that any proposer has

\footnotetext{
${ }^{20}$ The legal remedies that approximate this kind of protection are the enforcement of "no-compete" agreements and, to some respect, trade secret law. The applicability of trade secret law is not restricted to the development process per se. However, it has been noted (see Ordover (1991)) that it can provide protection in the process that leads to the application for a patent.

${ }^{21}$ The traditional protection against this kind of risk is the patent system.

${ }^{22}$ While in the case of a single innovator (i.e., $k_{0}=1$ ) no-compete clauses are able to guarantee this kind of protection, in the case of multiple innovators no-compete clauses only protect the innovator who makes the first offer.

${ }^{23}$ We limit the restitution to be allowed only when an innovator is not part of the firm because we want to allow agents to be able to write contracts that stand in court when IPR are enforced.

${ }^{24}$ Notice that the notion of Symmetry must be sightly readapted for this benchmark. In fact, since the payoff of a firm depend on the innovator being included or not, we must allow for offers to discriminate agents on the fact of their being the innovator or not. It is very simple to define a suitable notion of anonimity, where offers can
} 
to include the innovator in his offer to avoid the collection of the full value by the innovator after the formation of the firm. Therefore, the innovator is included in any offer made in equilibrium. This guarantees that the innovator appropriates $\pi_{1}=1 .^{25}$ It is easy to see that this result holds also in the case in which there are $k_{0}>1$ initial innovators. ${ }^{26}$

In the next section, we start analyzing imperfect property rights protection.

\section{The Partial Protection Regime}

In this section, we analyze the case in which patent protection is available, but information leakage can damage innovators during the development stage (i.e., the "Partial Protection" regime). In the model, this case can be represented by assuming that $\pi_{1}=1$ and $\pi_{2}=0$. In Proposition 1 , we characterize the unique SSPE for any initial set of innovators $K^{0}$. Then, in Corollary 2, we focus on the appropriation rate of a single innovator (i.e., $K^{0}=\{1\}$ ). All the proofs are presented in the Appendix.

Proposition 1 In the Partial Protection Regime, there is a unique SSPE. In this equilibrium each proposer always makes offers to one agent. In the subgames in which there are uninformed agents, proposers always choose to offer to an uninformed agent rather than an informed one.

Recall that we denote by $v_{k}$ the continuation value of an informed agent at a history $h \in H_{N}$ in which there are $k(h)$ informed players. To build the unique equilibrium sequence $\left\{v_{k}\right\}_{k=2}^{n}$ of the continuation values of each informed player, start with $n$ informed agents. When everybody is informed, symmetry guarantees that the continuation value of every player is $v_{n}=\frac{\delta}{n}$, i.e., $1 / n$-th fraction of the discounted pie $\delta$.

When $n-1$ agents are informed, consider the options of a proposer. He can either form a firm only with informed agents, or he can include an uninformed agent in his offer. If the offer includes discriminate only on the basis of agents being informed or not and on the basis of agents being the innovator or not.

${ }^{25}$ To see this, focus on a subgame in which everybody is informed, and let $\bar{v}$ and $\underline{v}$ be the maximum and the minimum continuation value sustainable in equilibrium for the innovator, respectively. Then, every other agent must have $\frac{1-\underline{v}}{n-1}$ and $\frac{1-\bar{v}}{n-1}$ as maximum and minimum continuation value, respectively. Observe that we have $\bar{v} \leq \frac{1}{n}\left(1-\frac{1-\bar{v}}{n-1}\right)+\frac{n-1}{n} \bar{v}$ and $\underline{v} \geq \frac{1}{n}\left(1-\frac{1-\underline{v}}{n-1}\right)+\frac{n-1}{n} \underline{v}$, which together imply $\underline{v}=\bar{v}=1$. Then, the innovator can always appropriate 1 by offering 0 to all the other agents in the initial offer.

${ }^{26}$ To see this, observe that in this case agents include all the innovators in the offers. Then, when everybody is informed, if the continuation value of the innovators sustainable in equilibrium are assumed to be in the range $[\underline{v}, \bar{v}]$, we have $\bar{v} \leq \frac{1}{n}(1-(k-1) \underline{v})+\frac{n-1}{n} \bar{v}$ and $\underline{v} \leq \frac{1}{n}(1-(k-1) \bar{v})+\frac{n-1}{n} \underline{v}$, which implies $\underline{v}=\bar{v}=\frac{1}{n}$. 
the uninformed agent, every agent has to be paid $\frac{\delta}{n}$. Suppose that he only offers to informed agents. Upon rejection, each of them is chosen as next proposer with probability $\frac{1}{n-1}$. In that event, each can guarantee themselves at least $1-\frac{\delta}{n}$ by making an offer to the only uninformed agent. Thus, the amount $\frac{\delta}{n-1}\left(1-\frac{\delta}{n}\right)$ represent a lower bound of the continuation value of an informed agent. However, as $\frac{\delta}{n-1}\left(1-\frac{\delta}{n}\right)>\frac{\delta}{n}$, it is always optimal to offer to the only uninformed agent. This implies that $v_{n-1}=\frac{\delta}{n-1}\left(1-\frac{\delta}{n}\right)$.

Working by backwards induction on the number of informed agents, assume that $k<n$ agents are informed, and the continuation value sequence is defined by $v_{h}=\frac{\delta}{h}\left(1-v_{h+1}\right)$ for all $h \in$ $\{k+1, . ., n-1\}$. Again, by making an offer to an informed agent, a proposer has to pay him at least $\frac{\delta}{k}\left(1-v_{k+1}\right)$, and it can be shown that $\frac{\delta}{k}\left(1-v_{k+1}\right)>v_{k+1}$. This implies that it is always optimal to make an offer to an uninformed agent rather than an informed one (i.e. information diffuses off the equilibrium path), and that $v_{k}=\frac{\delta}{k}\left(1-v_{k+1}\right)$. Moreover, since the sequence $\left\{v_{l}\right\}_{l=k+1}^{n}$ displays the property $m v_{k+m}>(m+1) v_{k+m+1}$ for any $m \geq 1$, it is not optimal to make an offer to more than one agent.

Let us now turn to the intuition behind the information diffusion. Focus on a proposer that has to choose between making an offer to either an uninformed agent or an informed one when $k$ agents are informed. By offering to an informed agent (or to a subset of the informed agents), the proposer forces that agent to choose between joining the firm or rejecting the offer and competing against $k$ other informed agents. By offering to an uninformed agent, the proposer makes him choose between joining the firm and competing against $k+1$ informed agents. Thus, uninformed agents face a fiercer competition upon rejection, and this tends to lower their continuation value. This effect tends to make uninformed agents "cheaper" to recruit than informed ones. On the other hand, after receiving an offer and becoming informed, by the inductive hypotesis, we know that an uninformed agent will have "cheaper" potential employees in the event that, upon rejection, he is chosen to make the next offer. This effect tends to increase an uninformed agent's continuation value with respect to the one of an informed agent. Proposition 1 shows that in equilibrium the first effect is stronger. Thus, it is optimal to hire uninformed agents, and in equilibrium more and more agents become informed off the equilibrium path.

At this point, one could be tempted to think that if the threat of future competition makes hiring uninformed agents cheaper, a proposer could profit from flooding the market by making offers to many agents. Proposition 1 shows that this is never profitable. By offering to many uninformed agents, one reduces their continuation payoffs but also promises a share to each of 
them. As the continuation values decrease with decreasing increments, it is not profitable to "flood" the market.

Proposition 1 allows us to compute the appropriation rate of a single innovator with an original idea, that faces the risk of information leakage. ${ }^{27}$ In the equilibrium presented in Proposition 1 , the innovator's appropriation rate is $1-v_{2}$. Since $v_{2}$ is a function of the number of agents in the game $n$ and of the discount factor $\delta$, we denote the appropriation rate $v(\delta, n) \equiv 1-v_{2}(\delta, n)$. We denote $v(1, n) \equiv \lim _{\delta \rightarrow 1} v(\delta, n)$ and $v(\delta, \infty) \equiv \lim _{n \rightarrow \infty} v(\delta, n)$.

Corollary 2 If there is a single innovator, $v(\delta, n)>\frac{1}{2}$ for all $n$ and $\delta$. Moreover, $v(\delta, \infty)=$ $\frac{1}{\delta}-\frac{e^{-\delta}}{\delta}$.

Observe that Corollary 2 implies that $v(1, \infty)=1-e^{-1} \simeq 0.632$, i.e. as the bargaining frictions disappear the innovator appropriates $63.2 \%$ of the value of his idea. This implies that the innovator enjoys an advantage that is not driven by legal enforcement but rather by equilibrium incentives. Corollary 2 suggests that the structure of the equilibrium described in Proposition 1 guarantees that the innovator's appropriation rate is not fully explained by the first-mover advantage.

To see how this result departs from the standard bargaining literature, consider what happens in our model when $n=2$. It is easy to see that in the only SPE, proposers always offer $\frac{\delta}{2}$, and agents always accept the offer. As the players get more patient ( $\delta$ tends to 1$)$, the first-mover advantage of the first proposer vanishes, and his payoff goes to $\frac{1}{2}$.

It is also possible to contrast Proposition 1 and Corollary 2 with a version of bargaining model in which information diffusion is possible, but does not influence the innovator's payoff. Consider the case in which there are $n>2$ agents. However, when an agent rejects an offer, he is the next proposer with probability 1 (when more than one agent simultaneously reject the same offer, each of them becomes the next proposer with the same probability). Consider an equilibrium in which proposers always offer $v=\frac{\delta}{1+\delta}$ to one agent. This is an equilibrium as $v$ satisfies $v=\delta(1-v)$. In this equilibrium the information diffusion does not play any role, and the payoff of the innovator is $1-\frac{\delta}{1+\delta}$, which is higher than $\frac{1}{2}$ only due to a first-mover advantage. In this equilibrium, making offers to uninformed agents is the same as making offers to informed agents since the competition among informed agents never arises.

In our case, we have that $\lim _{\delta \rightarrow 1} v(\delta, n)=1-\frac{1}{2}\left(1-v_{3}\right)>\frac{1}{2}$ for all $n$ and $\delta$. This is due to the presence of many agents and the competition arising upon rejection of any offer. In fact,

\footnotetext{
${ }^{27}$ The result can be easily related to the case of several initial innovators.
} 
off the equilibrium path, more and more agents become informed about the idea. This implies that upon rejection of an offer, the surplus is not going to be divided only among the current informed agents but among a larger set of agents. This effect works in favor of the innovator, as it lowers the continuation value of the agent he hires. We name $\frac{\delta}{2}-v_{2}$, which is the portion of the appropriation rate not explained by the first-mover advantage, the "information diffusion advantage."

\subsection{Information Leakage and Larger Firm Size}

The results presented so far rest on the assumption that only two agents are needed to develop an idea. If this is the case, innovators are able to appropriate a sizable share of the proceeds of their own ideas even in the absence of perfect IPR. One may wonder whether this prediction is robust with respect to a more demanding production technology. We model this by increasing the number of people required to develop an idea. When innovators need to hire many agents, they face the potential defection of all of them. Does this result in the total dissipation of an innovator's appropriation rate? Proposition 3 is a first step in this direction, since it generalizes Proposition 1 to larger firm size. All the proofs are in the Appendix.

Proposition 3 In the Partial Protection regime, if $m+1 \leq n$ agents are needed to develop an idea, there is a unique SSPE. In this equilibrium the proposers always make offers to $m$ agents. In the subgames in which there are uninformed agents, proposers always make offers to uninformed agents rather than informed ones.

Proposition 3 guarantees that the equilibrium structure when $m>1$ is similar to the case in which $m=1$ we studied before. Proposers always offer to exactly $m$ uninformed agents as long as the number of informed agent is less or equal to $n-m$. At histories where $k>n-m$, proposers offer to all the uninformed agents $\left(n^{U}=n-k\left(h_{i}\right)\right)$ and to the number of informed agents necessary to form the firm (i.e. $n^{I}=m-n+k\left(h_{i}\right)$ ). The equilibrium continuation value sequence $\left\{v_{k}^{m}\right\}_{k=2}^{n}$ is defined by $v_{n}^{m}=\frac{\delta}{n}$,

$$
v_{k}^{m}=\frac{\delta}{k}\left(1-(n-k) \frac{\delta}{n}\right)
$$

for $n-m \leq k<n$ and

$$
v_{k}^{m}=\frac{\delta}{k}\left(1-m v_{k+m}^{m}\right)
$$


for $2 \leq k<n-m$. One can show that $\left\{v_{k}^{m}\right\}_{k=2}^{n}$ is decreasing, which implies that it is optimal to hire uninformed agents rather than informed ones, as long as enough uninformed agents are left on the market. This allows the proposer to exploit the additional competition that will occur following a rejection. The temptation of flooding the market by offering to more than $m$ people in order to lower their continuation value turns out to be suboptimal as in the $m=1$ case.

Let us now focus attention on the case in which there is a single innovator and let us find his appropriation rate. Let $v(m, \delta, n) \equiv 1-m v_{m+1}^{m}$ be the innovator's payoff when $m+1$ agents are required to form a firm, $n$ is the number of agents in the game, and $\delta$ is the discount factor. Let $v(m, \delta, \infty) \equiv \lim _{n \rightarrow \infty} v(m, \delta, n)$.

Corollary 4 If $m+1<n$ agents are needed to develop an idea and there is a single innovator, $\lim _{m \rightarrow \infty} v(m, \delta, \infty)=e^{-\delta}>0$.

For very large $m$, and as the bargaining frictions disappear, the presence of the information diffusion advantage keeps the appropriation rate bounded away from zero. Observe that Corollary 4 implies that $v(\infty, 1, \infty)=e^{-1}$, i.e., the innovator's share as the number of agents required to develop the product grows to infinity is always larger than 0.368 . This implies that the innovator is able to appropriate a sizable share of profits even though he needs to hire many agents to develop the business idea. Again, this result is driven by the presence of an information diffusion advantage. In fact, consider an $m+1$-player version of our bargaining game. In the only SSPE, the first proposer appropriates $1-\frac{\delta m}{m+1}$. Note that as $\delta$ tends to 1 , the first mover advantage disappears, and the innovator appropriates $\frac{1}{m+1}$. For high $m$, the appropriation rate of the innovator goes to zero.

In our case, the innovator appropriates $v(m, \delta, n)>1-m \frac{\delta}{m+1}$. The difference $m \frac{\delta}{m+1}-m v_{m+1}$ is the information diffusion advantage for the $m>1$ case. Once again, the innovator benefits from the presence of information diffusion in equilibrium. In fact, information diffusion guarantees that upon rejection of an offer, informed agents anticipate some dissipation of the value to uninformed agents. This tends to decrease their continuation value and improves the position of a proposer. As the number of people required to form a firm is larger, information diffuses faster, and the information diffusion advantage becomes stronger.

This concludes the analysis of the Partial Protection regime. In the next section, we analyze the No Protection regime. 


\section{The No Protection Regime}

In the previous section we analyzed the Partial Protection regime in which patents were available at the end of the development process. Therefore, the first firm that formed enjoyed a monopoly on the final product market. In that case, the innovators' payoff was substantial due to the information diffusion that occurred off the equilibrium path.

We now analyze a situation in which patents are not available (i.e. No Protection regime), and the market can accommodate more than one firm. If this is the case, a monopoly is not guaranteed after the creation of the first firm. As a result, proposers face two problems when trying to form a firm. First, since the information gets transmitted while they bargain, they have to take into consideration the possibility of employees' defection during the development stage. Second, if they do not include all the informed agents in their firm, a second firm can be formed, and they will eventually face competition on the final product market. As a consequence, instead of earning the whole surplus of the idea, the first firm gets $\pi_{1} \leq 1$, and the second firm gets $\pi_{2} \cdot 28,29$

In this Section, we focus on two main issues. First, in this model, the market structure is determined endogenously in equilibrium. Whether we will observe monopoly or competition arising will depend on the way innovators solve their trade off between forming small firms and allowing for competition and forming large firms that preclude competition. In Subsection 4.1 we analyze the set of equilibria and discuss the market structure outcome. We provide conditions

\footnotetext{
${ }^{28}$ These profits should be interpreted as incorporating any downstream effects due to imitation. Observe that $1-\pi_{1}$ is a measure of the cost of competition for an incumbent firm. Recall that competition implies $\pi_{1}+\pi_{2} \leq 1$ and, since the first firm to form chooses the most profitable project, $\pi_{1} \geq \pi_{2}$. See Section 5 for a discussion of the case in which $\pi_{1}+\pi_{2}>1$.

${ }^{29}$ The second firm can produce a product identical to the first firm, or it can exploit the idea in some secondary application. To interpret the parameters $\pi_{1}$ and $\pi_{2}$, notice that our model is consistent with two different situations: first, consider the case in which there is no secondary application for the idea, and the second firm produces a product identical to the first firm. In this case, the two firms compete on the same market, so that $\pi_{1}$ and $\pi_{2}$ are both very small. If the two products are differentiated, $\pi_{1} \leq 1$ is due both by the decrease in the first market's size due to the presence of the second product and by some substitutability between the two products. The parameter $\pi_{2}$ measures the size of the market for the secondary application of the idea, and is also affected by the degree of substitutability between the two products (e.g., if the two firms play Bertrand competition, $\pi_{2}$ and $\pi_{1}$ are close to zero). $\pi_{1}$ close to 1 occurrs when the secondary application of the idea is is well-differentiated and relatively uninportant. $\pi_{1}$ and $\pi_{2}$ both close to $1 / 2$ may denote well-differentiated and equally important applications (e.g. consider the demand functions derived by Cobb-Douglas utility function $\left.u(x, y)=(x y)^{1 / 2}\right)$.
} 
on the parameters of the model for which competition is the market outcome and conditions for which monopoly arises. ${ }^{30}$

Second, as before, we are interested in the ability of a single initial innovator to appropriate the rents generated by his idea. In Subsection 4.2 we check whether patents are the crucial element in guaranteeing sizable appropriation rates to innovators.

At the heart of the analysis of this Section is the trade-off that a proposer faces between making an offer that include all the other $k\left(h_{i}\right)-1$ other informed agents (and generating a monopoly market outcome), and making an offer that does not include all of them (and generating a competition market outcome). We refer to the first kind of offer as "grand coalition" offer (i.e. an offer such that $\left.n^{I}=k\left(h_{i}\right)-1\right)$, and to the second kind of offer as a "cost minimizing" offer (i.e. an offer such that $n^{I}<k\left(h_{i}\right)-1$ ).

To simplify the exposition, throughout this Section we assume that $n$ is large. In the Appendix we present the results for the finite $n$ case.

\subsection{Equilibrium Market Structure}

We start the analysis of the equilibrium market structure by observing that if there is a single innovator monopoly is always the final market outcome.

Lemma 5 When there is a single innovator, the equilibrium market outcome is always a monopoly.

The intuition behind Lemma 5 is simple. When a single innovator makes the first offer, he can offer to the minimal number of agents he needs and still guarantee himself a monopoly. This implies that the innovator does not face the trade-off between a grand-coalition and a costminimizing offer, and will always form a monopoly at minimum cost.

Once we start from a larger set of innovators monopoly cannot be guaranteed. When there are several innovators, the trade-off between 'large', monopolistic firms and 'small', competitive firms begins to bite. Proposers must decide which path they prefer to follow. Grand coalition offers involve a larger pie to share but a relatively large number of employees to divide it among. Alternatively, the proposer can make a cost-minimizing offer, that is, an offer that focuses on forming a firm at the minimal cost, but generates market competition. Therefore, this option entails a smaller pie to be shared but also a lower total value to be paid to the employees.

\footnotetext{
${ }^{30}$ The normative implications of these results are differed to section 5 .
} 
Note that in all SSPE, if the number of informed agents is high enough, a proposer can guarantee himself almost $\pi_{1}$ by making a cost minimizing offer. To see this, notice that in any SP Equilibrium and any subgame $h \in H_{N}$, the sum of the continuation values of the informed agents cannot be larger than $\delta$, i.e., $\sum_{i \in K(h)} v^{i}(h) \leq \delta .{ }^{31}$ If the strategies are symmetric, the continuation values of all the informed players must be equal. Because the pie is bounded in size, this means that when there are many informed agents, the continuation value of each agent must converge to zero. In turn, this implies that whenever an agent is called to make an offer, he can guarantee himself almost $\pi_{1}$ by offering to one agent his continuation value.

Proposition 6 offers conditions under which competition arises as equilibrium market outcome. The proofs of Propositions 6 and 7 are presented in the Appendix.

Proposition 6 If $\pi_{1} \geq \frac{1-\delta^{2} \pi_{2}}{1+\delta}$, there exists a $\bar{k}$ such that for any subgame with $k \geq \bar{k}$ informed agents, there is a unique SSPE. In this equilibrium proposers always make offers to one uninformed agent. As a result, competition is the only equilibrium market structure outcome.

Proposition 6 establishes sufficient conditions for agents to make only cost-minimizing offers. If agents make cost-minimizing offers, competition is the equilibrium market structure outcome. When there are many informed agents in a negotiation subgame, the cost of a grand coalition offer must be close or above $\delta\left(\pi_{1}+\delta \pi_{2}\right)$, as this is the minimal (discounted) pie that is going to be shared upon rejection. Therefore, by offering a grand coalition offer, one can expect to receive at most $1-\delta\left(\pi_{1}+\delta \pi_{2}\right)$. On the other hand, by making a cost-minimizing offer the proposer can secure a payoff of almost $\pi_{1}$. Thus, if $\pi_{1}>1-\delta \pi_{1}-\delta^{2} \pi_{2}$ (i.e., if $\pi_{1}>\frac{1-\delta^{2} \pi_{2}}{1+\delta}$ ), no grand coalition is offered for high $k$.

Proposition 6 implies that if the degree of potential market competition is low and the number of informed agents is high enough, competition is always the equilibrium market outcome. Notice that since $\pi_{1}+\pi_{2} \leq 1$, this prediction is in contrast to bargaining efficiency, which would require monopoly to arise. The source of this inefficiency is that proposers are restricted in their strategies. Proposers are forced to offer to all the informed agents if they want to sustain a monopoly. When the number of informed agents is high and the difference between monopoly rents and $\pi_{1}$ is low, proposers may find it optimal to generate competition instead.

\footnotetext{
${ }^{31}$ This follows trivially from the fact that at any history $h \in H_{N}$, the informed agents are dividing a discounted pie that is smaller or equal to 1.
} 
The following Proposition relates to the case of high levels of potential market competition, i.e. low $\pi_{1}$.

Proposition 7 If $\pi_{1}<1-\delta$, there is a unique SSPE. In this equilibrium proposers always make grand coalition offers. Therefore, the equilibrium market outcome is always a monopoly.

The intuition behind Proposition 7 is the following. The cost of making a grand-coalition offer never exceeds $\delta$. Therefore, a grand coalition offer always guarantees a payoff of at least $1-\delta$. On the other hand, a cost-minimizing offer guarantees at most $\pi_{1}$. Therefore, if $\pi_{1}<1-\delta$, no agent would ever make a cost-minimizing offer, no matter how many informed agents there are on the market.

Proposition 7 guarantees that if $\pi_{1}$ is low, monopoly always arises. This implies that no matter how many innovators are initially aware of the new idea, information remains confined within the boundaries of one firm.

The last result has an important implication about information diffusion. Proposition 7 shows that if $\pi_{1}$ is low, offers never include uninformed agents, i.e. $n^{U}=0$, and grand coalition offers are made only to informed agents. This implies that information diffusion never occurs on or off the equilibrium path. In the next Section we analyze how this feature of the equilibrium relates to the innovator's appropriation rate.

Next, we check the equilibrium outcomes for the middle range of potential market competition, i.e., $\pi_{1} \in\left(1-\delta, \frac{1-\delta^{2} \pi_{2}}{1+\delta}\right)$. Proposition 8 shows that in this range there are multiple equilibria and that one can sustain both competition and monopoly market outcomes in equilibrium.

Proposition 8 If $\pi_{1} \in\left(1-\delta, \frac{1-\delta^{2} \pi_{2}}{1+\delta}\right)$, there is a $\bar{k}$ such that for all subgames with $k>\bar{k}$ informed agents, there exist both a SSPE in which competition is the market outcome and an equilibrium in which monopoly is the market outcome.

To give the intuition for this result consider an equilibrium in which competition arises. One can sustain competition in every subgame using the following strategies. As long as proposers make cost-minimizing offers, the continuation game involves future cost-minimizing offers. This entails a large share of the profits to the proposers. But proposers may be tempted to go for a grand-coalition offer. Such behavior is discouraged by the threat that if such offers are made, continuation games will involve further grand coalition offers that, as we will see in the next Subsection, tend to increase the continuation value of the employees. 
In the next section we analyze the implication of these results to the innovators' appropriation rates.

\subsection{Appropriation Rates in the No Protection Regime}

In Section 3, we highlighted the presence of the information diffusion advantage, that enabled innovators to appropriate sizable shares of the profits. However, from Proposition 7, we know that if the additional threat of potential market competition is added, sometimes information diffusion disappears in equilibrium. Proposers may prefer to preempt the threat of market competition by making grand coalition offers that, in equilibrium, do not involve any uninformed agent. One may wonder to what extent the disappearance of information diffusion affects the innovator's payoff.

On the other hand, in the absence of patents, it seems that the threat of market competition may serve to strengthen the bargaining power of innovators. ${ }^{32}$ In this Subsection, our aim is to check how these effects relate to the appropriation rate of a single innovator. ${ }^{33}$

We start the analysis with an observation: if exactly two agents are informed, the continuation value of each of them is at most $\frac{\delta}{2}$. This implies that the innovator can always secure $1-\frac{\delta}{2}$ by offering $\frac{\delta}{2}$ to one agent. ${ }^{34}$

In Proposition 9, we show that, for low levels of potential market competition, the unique SSPE is similar to the equilibrium we characterized in Section 3. Recall that $v(\delta, \infty)$ is the appropriation rate of a single innovator for high $n$.

Proposition 9 If there is a single innovator, there exists a $\widetilde{\pi} \in\left[\frac{1-\delta^{2} \pi_{2}}{1+\delta}, 1\right)$ such that, if $\pi_{1} \geq \tilde{\pi}$, there is a unique SSPE in which (i) proposers always make offers to one uninformed agent, and (ii) $v(\delta, \infty)>1-\frac{\delta}{2}$, and $v(\delta, \infty)$ is decreasing in $\pi_{1}$ and $\pi_{2}$.

Proposition 9 implies that the equilibrium characterized in Section 3 holds for a range of values of $\pi_{1}$. Let us now look more carefully at the appropriation rate of the innovator in this equilibrium.

\footnotetext{
${ }^{32}$ Anton and Yao (1994) show, in a related model, that the stronger the potential competition on the market, the higher the appropriation levels of innovators may be.

${ }^{33}$ It is easy to relate the following results to the case of sevelar initial innovators.

${ }^{34}$ Note that this is a result of the assumption that only one agents is needed in the production function. When the production function involves more and more agents, the minimum payoff an agent can secure tends to $1-\delta$.
} 
Notice that the generic element of the sequence $\left\{v_{k}\right\}_{k=2}^{n}$ for large $n$ is

$$
\begin{aligned}
v_{k}= & \pi_{1}\left[\sum_{i=1}^{\infty} \frac{(-1)^{i-1} \delta^{i}(k-1) !}{(k-1+i) !}+\sum_{i=1}^{\infty} \frac{\delta^{i}}{k}\right] \\
& \left.\left.+\pi_{2} \sum_{i=1}^{\infty}(k+i) \sum_{j=1}^{\infty} \delta^{j} \frac{(k+i) !}{(k+i+j) !}(-1)^{j-1}\right)\right)
\end{aligned}
$$

The innovator's appropriation rate is given by

$$
v(\delta, \infty)=1-v_{2}>1-\frac{\delta}{2}
$$

The innovator's ability to guarantee a share larger than $1-\frac{\delta}{2}$ is now due to two effects. The first effect, the information diffusion advantage, was introduced in Section 3. As the equilibrium involves information diffusion, the innovator enjoys the lower continuation value of the employee he offers to. This effect allows the innovator to be able to appropriate more than half of the surplus of the continuation game, i.e. $\pi_{1}+\delta \pi_{2}$.

But the innovator is succeeding in getting even a higher share. The innovator is able to appropriate also the entire gap between monopoly profits and the surplus under competition, i.e., $1-\left(\pi_{1}+\delta \pi_{2}\right)$. The innovator is able to do that as he can successfully threaten a potential employee that if he rejects, the result will be market competition. We term this new effect the "threat of competition advantage".

To understand the "threat of competition advantage", notice that the continuation value of the potential employee is increasing in both $\pi_{1}$ and $\pi_{2}$. When competition arises in any subgame, the total pie that is divided among agents is $\pi_{1}+\delta \pi_{2}$. Therefore, as this pie gets larger, agents' continuation values increase. On the other hand, the appropriation rate of the innovator is decreasing in $\pi_{1}$ and $\pi_{2}$. As $\pi_{1}$ and $\pi_{2}$ decrease, the threat of competition in the subgame that ensues in case of a rejection becomes more powerful. This enables the innovator to pay less to his employees and therefore increase his own share of the profit.

The link between fiercer potential competition on the market and higher bargaining power of the innovators is very intuitive. In a different model, Anton and Yao (1994) consider this effect and show that fiercer competition on the market cannot hurt the innovator and often strengthens his position. $^{35}$ The main difference between the two models is that, in Anton and Yao, the interaction

\footnotetext{
${ }^{35}$ Anton and Yao (1994) analyze a one-period model in which an inventor needs a manufacturer to develop his business idea. He can exploit the threat to reveal his idea to a second manufacturer to increase is payoff. In particular, this is the case when the inventor has all the bargaining power in the negotiation with the manufacturers.
} 
is modeled as a one shot, take-it-or-leave-it game. Our approach relies on the observation that once the manufacturer knows the idea, he can still suffer from the defection of his own employees and face an information leakage problem himself. This suggests a bargaining game with a different protocol, and, as a result, the threat of competition in the continuation game could end up not being credible. The credibility of the threat of competition is explored in Proposition 9 and in the next two results. In particular, Proposition 9 shows that for low degrees of potential market competition, the threat of competition is credible and the innovators does in fact exploit it.

In Proposition 10 we show that for high degrees of potential market competition, the threat of competition ceases to be credible. As a result, the innovator's position is compromised, and he is unable to appropriate the gap between the monopoly and competition rents.

Proposition 10 If there is a single innovator, and if $\pi_{1}<1-\delta$, we always have $v(\delta, \infty)=1-\frac{\delta}{2}$.

Proposition 10 has implications on the employees' wages. Notice that the firm that arises in this equilibrium demands a high wages for the employee. This is because, in the equilibrium described in Proposition 7, informed agents are always included in the grand coalition offers made in equilibrium. This tends to increase their continuation values, and depress the innovator's appropriation rate even further. ${ }^{36}$

As already mentioned in Subsection 4.1, for intermediate values of $\pi_{1}$, there are multiple equilibria. Our final result shows that for values of $\pi_{1}$ in $\left[1-\delta, \frac{1-\delta^{2} \pi_{2}}{1+\delta}\right]$ one can sustain equilibria in which the innovator appropriates the minimum appropriation rate, $1-\frac{\delta}{2}$, as well as equilibria in which he is able to appropriate more than $1-\frac{\delta}{2}$.

Proposition 11 If there is a single innovator and if $\pi_{1} \in\left[1-\delta, \frac{1-\delta^{2} \pi_{2}}{1+\delta}\right]$, there exist both a SSPE in which $v(\delta, \infty)=1-\frac{\delta}{2}$ and a SSPE in which $v(\delta, \infty)>1-\frac{\delta}{2}$.

\footnotetext{
${ }^{36}$ For the case of $k^{0}>1$ initial innovators, "grand coalition" firms arise and they demand high wages for the employees. Note that all the employees of the firm are guaranteed strictly positive shares of the profits, even though their marginal contribution to the firm's production is zero. This is because the agents are paid not according to their productivity but rather accordinlg to the information they hold. Observe that if we compare the wage an employee earns in the firm that forms in equilibrium (i.e. $\left.\frac{\delta}{k^{0}}\right)$ with the wage that an employee earns in the firm that forms in equilibrium in a Partial Protection regime (i.e. $v_{k^{0}+1}$ ), we get that $\frac{\delta}{k^{0}}>v_{k^{0}+1}$. The difference $\frac{\delta}{k^{0}}-v_{k^{0}+1}$ is explained by the fact that if there are no patents and $\pi_{1}$ is low, an informed agent is always included in the grand coalition that forms upon his rejection. This result is in line with interesting empirical findings by Kumar, Rajan and Zingales (2001), who, in a cross-section and cross-country analysis on firm size, show that one of the explanatory variables of larger firm size is the presence of high-wages in the industry.
} 
In the middle range, a competitive market outcome is sustainable only if the number of informed agents is high enough. However, Proposition 11 shows that there are equilibria where a single innovator can still benefit from it. In fact, in the equilibrium we present in the Appendix, the innovator's payoff is higher than $1-\frac{\delta}{2}$ even if a competitive market outcome can be sustained only for a high enough number of informed agents. The innovator's success is again due to the information diffusion and the threat of competition advantages.

The analysis carried out in this section highlights that, in the absence of legal protection, innovators' appropriation rates are still protected by equilibrium effects. In particular, whenever $\pi_{1}>1-\delta$ there exist equilibria that provide the innovator with appropriation rates that are bounded away from the lower bound, i.e. $1-\frac{\delta}{2}$.

\section{Discussion and Welfare Implications}

\subsection{Modelling Assumptions}

Information Leakage. An important feature of our model is the presence of information leakage, which is captured by assuming that developing an idea requires collaboration and that collaboration entails information sharing. The first of these assumptions is motivated by a production function increasing in labor. Involving more people in the development stage may increase the productivity and quicken the development process. The second assumption is motivated by the incentives of innovators to inform their employees. Information is an input in the development. The more information is shared with co-workers, the more efficient the development stage may be.

Therefore, the innovator faces a trade-off. On one hand, he would like to collaborate with a number of other agents and inform them. On the other hand, he has an incentive to hold information back as he fears information leakage and employee defection. Innovators will often solve this trade-off by collaborating with some agents and sharing information to some extent.

In this paper we abstract from the above trade-off by assuming that innovators must hire a minimal number of agents, and must share all the information with them. These assumptions simplify our analysis and allow us to focus attention on innovators' appropriation rates. As our results are positive from the point of view of innovators, weakening these assumptions would only strengthen our results.

The assumption of information sharing should not be interpreted in the sense that all the 
information is exchanged immediately at the time of an offer. We prefer the point of view that contracts on undeveloped ideas do not become immediately binding. However, there is a moment in which they do. This moment occurs when the idea becomes well defined and when someone defecting from the original team can still successfully compete with the other informed agents in the development process. We think about the situation in which an agent can defect at any time between the moment in which they learn the information and the moment in which the contract becomes binding.

Bargaining Protocol. The bargaining protocol we use in this paper is a natural benchmark of the applied situation we address. The symmetry of the probabilities with which nature chooses the next proposer captures the symmetry among the informed agents. Once an agent is made aware of the information, nothing differentiates him from the inventor anymore. ${ }^{37}$ This implies that every informed agent should have the same probability of being the next proposer. ${ }^{38}$

In our model, when an agent receives an offer, he is invested with two distinct abilities: he perfectly learns the idea and he receives veto power on the success of the offer itself (i.e., the offers are conditional upon the acceptance of all the agents included in them). It is interesting to discuss the consequences of altering these assumptions by weakening the position of the recipient of an offer. However, we want to stress that, since the results in this paper show that in the absence of perfect IPR innovators are still protected by equilibrium effects, improving the position of the innovator always results in strengthening the point of this paper. The consequences of decreasing the recipients' ability to understand the idea are discussed in an extension of the model presented in Section 5.4.

Relaxing the second assumption amounts to consider unconditional offers. If offers are unconditional, the acceptance of one agent is enough for the offer to be binding and a firm to form. We

\footnotetext{
${ }^{37}$ For symmetric offer probabilities see also Baron and Ferejohn (1989). One could think about an extension in which the inventor has a deeper understanding of the idea that gives him an advantage in the competition in being the next offerer, and from there to be chosen by nature with higher probability. Besides increasing the appropriation rates of the inventor, we conjecture that this would not change the quality of the results.

${ }^{38}$ One could also think of a different model in which once an offer is rejected, all the informed players can make simultaneous offers. We do not think that this would add qualitative insight to our analysis. Such a model would have to specify tie-break rules (e.g. to assign the profit if more than one firm are formed simultaneously or to resolve situations of players making simultaneous offers to each other). Then, at least in a partial-protection regime, the presence of an increased competition advantage of the innovator could be conjectured, as uninformed agents still face fiercer competition upon rejection.
} 
find these kinds of offers unfit for our applied situation because we want to capture the competition that arises among the informed agents once they all know the information. In order to do so, we give the possibility of counter-offers to all the informed agents.

In a version of our model in which $\pi_{1}=1, \pi_{2}=0$ and unconditional offers are possible, consider the situation in which the innovator offers zero to two agents, and they both accept. This is an equilibrium, since none of the agents is pivotal in the formation of the firm, but the agents are not given the chance to make a counter-offer after they learn the idea. ${ }^{39}$ Notice also that this assumption is consistent with other models in the literature on intra-firm bargaining: both Stole and Zweibel (1996) and Wolinsky (2000) assume renegotiation of all contracts once an agent has defected from the firm.

Market competition. For simplicity, in our model the market can accommodate at most two firms. It is possible to relax this assumption and allow the final product market to accommodate any number of firms. As the number of firms increases, the competition gets fiercer, and the profits of all the pre-existing firms decrease. ${ }^{40}$ This extension does not change the quality of our results. This is because the proposers still face the trade-off between a grand-coalition offer that would guarantee a monopoly and a cost-minimizing offer that would allow competition to emerge. The potential competition may be more harmful for the first firm, as in competition it can expect to get at most $\pi_{1}$. This increases the incentives to make grand-coalition offers, and expands the range of parameters for which we expect grand-coalition to emerge.

In the presentation of the model, we restrict our attention to the case in which $\pi_{1}+\pi_{2} \leq 1$, i.e. the presence of a second firm can only generate competition and dissipate some of these rents. Relaxing this assumption and allowing for the case $\pi_{1}+\pi_{2}>1$ requires some frictions to prevent the first firm from developing both applications. ${ }^{41}$ However, the analysis of the $\pi_{1}+\pi_{2}>1$ case yields to similar results. ${ }^{42}$

\footnotetext{
${ }^{39}$ In turn, this leads to a lower bound for the innovator's payoff in the no protection regime: the innovator has now the possibility to offer 2 agents and get $1-2 \delta \pi_{2}$. For low values of $\pi_{2}$, this is an improvement with respect to the bound $1-\frac{\delta}{2}$. The results on endogenous market structure display a trade-off between grand-coalition or cost-minimizing offers similar to the one present in the model we analyze.

${ }^{40}$ All the possible profits are represented by a triangular $m \times m$ matrix $\Pi$, such that the generic element $\pi_{i, j}$ is the profit of firm $i$ if $j$ firms are on the market. We have $\pi_{i, j}>\pi_{i, j+1}$ and $\pi_{i, j} \geq \pi_{i+1, j}$ for all $i, j$. Moreover, increased competition lowers the sum of the profits, i.e. $\sum_{i \leq j} \pi_{i, j}>\sum_{i \leq j+1} \pi_{i, j+1}$.for all $i, j$.

${ }^{41}$ E.g. timing problems, necessity to specialize, increasing management costs etc.

${ }^{42}$ In particular, if $\pi_{1}+\pi_{2}>1$, it can be easily checked that when all the $n$ agents are informed a grand coalition offer costs at least $(n-1) \frac{\delta}{n}$. Then, whenever $\pi_{1}>1-\delta$, for a high enough number of informed agents competition
} 
Equilibrium concept. The definition of SSPE given in the Appendix requires two conditions to be satisfied. First, the distributions of offers used by the players have to be anonymous. Second, given the anonymity of the offers, symmetry requires that when nature chooses the next proposer from among all the informed players, every potential proposer is expected to behave in the same way. Notice that in every subgame starting with nature about to choose the next proposer, every potential proposer faces a different set of other informed players and then a different action set. This makes it impossible for a symmetric strategy profile to include offers that discriminate informed players on the basis of their identity. This is the reason why, in order to establish a notion of symmetry, we first need strategies to be anonymous with respect to the other players.

Focusing the attention to SSPE simplifies the analysis by making the computation of the equilibrium continuation values sequence tractable. Enlarging the set of equilibria to all the Subgame Perfect equilibria is likely to generate a high level of indeterminacy in the predictions of the model. For instance, in the Partial Protection regime, enlarging the set of equilibria to all the SPE leads to a result in which both almost full appropriation and almost no appropriation can be supported in equilibrium when agents are very patient.

The focus on SSPE allows us to highlight the strategic aspects that are specific to our bargaining model in contrast to other models of multi-agent bargaining. Our analysis introduced a novel equilibrium effect that is driven by the endogeneity of the set of potential proposers. We have shown that the implication of this effect was an asymmetry in the equilibrium payoffs that holds even as bargaining frictions disappear.

A possible alternative to SSPE could have been to require equilibria to be Stationary with respect to the number of informed agents. Although most of the predictions of our analysis remain the same under this alternative equilibrium concept, we believe that not allowing strategies to depend on histories is too restrictive to uncover all the strategic insights the model produces. ${ }^{43}$ Moreover, we conjecture that for a substantial range of parameters, Stationary equilibria may not exist for an infinite- $n$ version of the model. ${ }^{44}$

arises. Notice that the case $\pi_{1} \leq 1-\delta$ is not consistent with the assumption $\pi_{1} \geq \pi_{2}$ in the case in which $\pi_{1}+\pi_{2}>1$. This implies that for high enough $k$ we always have a competitive market outcome. However, for lower $k$ we could still have monopolistic market outcomes.

${ }^{43}$ It can be shown that the only SSPE found in Proposition 1 is also the only Stationary SP equilibrium in the partial protection regime. Also, in the no protection regime, we find grand-coalition offers for high degrees of competition, and cost-minimizing offers for low degrees of competition under Stationary SP equilibria.

${ }^{44}$ In particular, we conjecture this happens for $\pi_{1} \in\left[1-\delta, \frac{1-\delta^{2} \pi_{2}}{1+\delta}\right]$. 
Matching heterogenous agents. In our model, we assumed that agents are homogeneous and that the only element that differentiates them is the knowledge of the idea. An interesting extension of our model is to consider a market populated by two types of agents, say type A and type B (e.g. engineers and venture capitalists). A firm must be formed by an assortative match of these two types. It is easy to show that our results in the partial protection regime extend to this situation. ${ }^{45}$ This suggests an important implication of our model on the incentives to finance new ideas. We differentiate between the moment in which an idea arises and the beginning of its development. While the process of financing innovation was traditionally seen as an up-front investment followed by a research stage, this view has changed dramatically in the recent past. After getting a new idea at a relatively low cost, inventors now search for investors to finance its development. Our results suggest that the presence of information leakage increases the share appropriated by a venture capitalists, so that a weak protection of IPR improves the return of the investment in the development of new ideas. In fact, even in the presence of a competitive market for venture capitalists, the presence of information leakage increases the bargaining power of venture capitalists and increases their appropriation rate with respect to a perfect protection legal regime.

Stochastic information leakage. Throughout the analysis, we assumed that the act of hiring/developing necessarily involves full sharing of the information about the idea. Obviously, this is a strong assumption. Organizations find ways to secure information against insiders as well as against outside intruders. Information is often classified, and different agents gain access to different pieces of information. These measures will tend to decrease the amount of information the innovator must share with potential employees. But as long as these measures are costly, some information will always leak. One way to model such an extension is to assume that there is a probability $\alpha$ with which an agent who is made an offer learns the idea. Again, we expect our qualitative results to hold. The SSPE in the partial protection regime is still unique and

\footnotetext{
${ }^{45}$ Assume that there are $n$ agents of type $\mathrm{A}$ and $n$ agents of type $\mathrm{B}$ and that a type-A agent is the first to make an offer. It is easy to see that the quality of our results remains intact. In fact, in a partial protection regime, suppose that all $2 n$ agents are informed. Then, symmetry guarantees that the continuation value of each agent is $\frac{\delta}{2 n}$ regardless the type. Suppose now that there is one type-A agent uninformed. If a type A is chosen to make an offer, he can just hire an informed type B agent for $\frac{\delta}{2 n}$. On the other hand, if a type B is chosen to make an offer, by hiring an informed, he has to pay at least $\frac{\delta}{2 n-1}\left(1-\frac{\delta}{2 n}\right)$, while offering to an uninformed, he has to pay just $\frac{\delta}{2 n}$. Working backward, we can build the unique SSPE in which uninformed agents are always made offers to when available.
} 
the payoff for the innovator higher than the one of the partner. For high $n$, the payoff to the innovator is $\frac{1}{\alpha \delta}\left(1-e^{-\alpha \delta}\right)$. which is higher than the payoff to the innovator when there is full information sharing. Moreover, the innovator's appropriation rate is decreasing in $\alpha$ and reaches full appropriation when $\alpha$ approaches zero.

Unstable offers. In our model, we assumed that once a firm forms, it is stable and it completes the development of the product. An alternative modelling assumption can be made. In particular, we can assume that once a firm forms, it breaks down with some exogenous probability $\alpha>0$. In this case, the pie to share in the next period becomes $\frac{1-\alpha}{1-\delta \alpha}$ in the monopoly case, $\left(\pi_{1}+\delta \pi_{2}\right) \frac{1-\alpha}{1-\delta \alpha}$ in the competition case. The results in our paper are robust for low $\alpha$. In particular, uninformed agents are offered to, and an information diffusion advantage protects the innovator's payoff. This extension is interesting since in equilibrium information diffusion arises on the equilibrium path.

Cost of transmitting information. Our results are robust to the extension in which it is costly to transmit information. In particular, assume that making an offer to an uninformed agent involves some training, or time spent in explanations, that translate in some cost $c>0$. Making an offer to an informed agent allows to save such cost. This modification implies that, when a high enough number of people are informed, proposers will stop making offers to uninformed agents, even if some will still be present. However, as long as $c$ is small enough, an information diffusion advantage is present and protects the innovator's payoff. Qualitatively, the results of the No Protection regime are also unchanged.

\subsection{Welfare Implications}

A vast literature analyzes the use of IPR to increase the incentives to innovate. ${ }^{46}$ We suggest that the effect of patents on the incentives to innovate must be examined more carefully and that the importance of IPR may have been overvalued. In estimating the trade-off between the harms and benefits of IPR, too much weight was given to the benefit side in providing incentives to innovate.

This overestimate is due to the fact that the strategic aspects of information diffusion have not been taken into consideration. Our analysis of the Partial Protection regime suggests how information leakage in the development process of a product affects the appropriation rate of an

\footnotetext{
${ }^{46}$ The literature on patents is very large. Just to mention the main papers that opened this field, see Schumpeter (1943), who rasied the claim that monopoly is necessary to encourage R\&D, and Arrow (1962), who addressed the question of how much the firm gains from innovation if a life-long patent is available.
} 
innovator. As a consequence, in the absence of perfect protection of IPR, the innovator's payoff is protected by the information diffusion advantage.

Our analysis of the No Protection regime suggests that incentive to innovate do not disappear when patents are not available. ${ }^{47}$ In particular, we showed that monopoly can still arise with no IPR. This outcome protects innovators from high degrees of potential market competition, and allows to identify a lower bound for innovators' appropriation rates. For lower degrees of potential market competition the innovator's payoff is still protected by the information diffusion advantage. Moreover, it is also the case that the potential market competition may allow innovators to enjoy an additional increase in payoff due to the threat of competition advantage.

Another normative point that we derive from the No Protection regime regards the endogenous market structure analysis. Consider the monopoly market outcome that arises for high degrees of potential market competition. There are several reasons that suggest that such an outcome is not socially desirable. First, a monopolistic outcome from the point of view of consumers, is indistinguishable from the introduction of a patent. Second, the monopoly outcome is based on information remaining confined within the boundaries of the firm. This implies that this outcome precludes any information disclosure. This is not socially desirable as information disclosure may stimulate incremental research and future discoveries. ${ }^{48}$

Finally, from the point of view of the innovators' incentives, we showed that when monopoly is the market outcome the appropriation rate is the lowest possible. This is because the innovators have to bear high costs to recruit all the informed agents, that in this equilibrium have high continuation values. This suggests that departing from the No Protection regime to introduce narrow patents should improve social welfare. This is because narrow patents capture the situations in which the second firm markets a product very similar to the first one (i.e. low $\pi_{1}$ ), but do not apply to lower degrees of market competition, where the social costs of patent introduction may be higher. ${ }^{49}$

\footnotetext{
${ }^{47}$ See Boldrin and Levine (2002) for a similar conclusion drawn from a different perspective.

${ }^{48}$ It has been observed that when one firm is the exclusive user of a new technology (either because of secrecy or because of a patent), cumulative research may end up being discouraged. See Scotchmer (1991) and Scotchmer (1999). This argument usually relies on the assumptions that either frictions or specialization prevent the first patent-holder from fully exploiting all the applications of his patent.

${ }^{49}$ If the degree of potentail market competition is low, we can have a competitive market outcome. First, this is desirable for the consumers. Second, it allows for information disclosure as agents do not have incentives to keep information secret. This may stimulate incremental reseach. Finally, for high $\pi_{1}$, the appropriation rates of innovators are high and their incentives to innovate are relatively protected.
} 
The legal literature has suggested that innovation still takes place in the presence of weak enforcement of IPR. In particular, in contrast with common wisdom, Hyde (2000) points out that the Silicon Valley phenomenon cannot be understood without considering the weak IPR enforcement guaranteed by the State of California. ${ }^{50}$ Hyde's claims are based on the assumption that when IPR are weak, information diffusion occurs. Therefore he does not take into account the incentives of firms to protect themselves against information leakage.

In contrast our model takes account of the incentives innovators have to protect their information. We derive the conditions under which Hyde's claim holds. In particular, given California law, it is possible to make a case that the high-tech industry in Silicon Valley fits our No Protection regime. This industry is also characterized by a very fast growth of the markets, by a constantly increasing number of applications of high-tech ideas, and by a geographical concentration that facilitates communication. These facts suggest that $\pi_{1}, \pi_{2}$, and the number of agents initially aware of new ideas are all high. Our results show that in these cases competition will arise. This implies that information diffusion may occur, ideas are likely to be fully exploited and disclosed to stimulate future incremental research.

We explore the relation between IPR and industry performance further in Baccara and Razin (2001). This project's aim is to explore the conjecture that when a two-sided investment is required from both "employer" and "employee," a weak protection of IPR and the presence of information leakage may improve efficiency. It is well known that indivisible property rights and incomplete contracts generate inefficiency when the employees are required to exert a noncontractable effort in the production process. In fact, as an employee fails to appropriate the full value generated by his effort, his incentives to exert it are destroyed. It has been suggested by Aghion and Tirole (1994) and Lester and Talley (2001).that different allocations of property rights could help to improve this inefficiency as they provide more incentives for the employees. However, these papers take an optimal contract approach and compare some exogenously given property rights allocations.

In Baccara and Razin (2001), we argue that when IPR are weak, the presence of information leakage itself endogenously delivers a more equal division of the value and thus improves efficiency. To see this, consider an example in which a non-contractable effort from two agents is required to

\footnotetext{
${ }^{50}$ California prohibits no-compete clauses (unless one of a number of exceptions are met). The application extent of patents in software industry is also an issue that does not seem to have found a precise legal answer yet (see Bensen and Raskind (1991)).
} 
carry out the development of a product. Assume that the production function is $V(a, b)=(a b)^{\frac{1}{2}}$, where $a, b \in \Re_{+}$are the efforts of the employer and employee, respectively. The cost of effort is the same for both agents involved in the production, and it is defined by $c(e)=\frac{e^{2}}{2}$, where $e=a, b$. The first best effort levels $(a, b)$ maximize $V(a, b)-\frac{a^{2}}{2}-\frac{b^{2}}{2}$ and they are $a=b=1 / 2$. Without the presence of information leakage (when IPR are perfectly enforced), we have that if $x \in[0,1]$ is the share of the employee, the employer's effort is $a(x)=K^{\prime}(1-x)^{\frac{3}{4}} x^{\frac{1}{4}}$ and the employee's one is $b(x)=K^{\prime} x^{\frac{3}{4}}(1-x)^{\frac{1}{4}}$ for some positive constant $K^{\prime}$. By solving the employer's problem, it is easy to see that he offers the employee the share $x^{*}=1 / 4$.

Consider now the case in which there is information leakage, and focus on an example in which $n=3$. Let us consider a strategy profile in which proposers always offer an uninformed agent the minimum between his continuation value (i.e., $v_{k}$ ) and $1 / 4 .{ }^{51}$ When everybody is informed about the idea (i.e. $k=3$ ), the employer has to pay an employee at least his continuation value upon rejection $v_{3}$, which is the third part of the total value of the pie, $V\left(a\left(x_{3}\right), b\left(x_{3}\right)\right)-c\left(a\left(x_{3}\right)\right)-$ $c\left(b\left(x_{3}\right)\right)$, discounted by $\delta$. The employer has to find a share $x_{3} \in[0,1]$ that solves the following maximization problem

$$
\begin{aligned}
& \max _{x} K^{\prime \prime}(1-x)^{\frac{3}{2}} x^{\frac{1}{2}} \\
& \text { s.t. } V(a(x), b(x))-c(b(x)) \geq v_{3}
\end{aligned}
$$

where $K^{\prime \prime}$ is a positive constant. In equilibrium, the constraint of (1) is satisfied as long as $x_{3} \geq \frac{\delta}{3}$. We have that if $\frac{\delta}{3}<\frac{1}{4}$, the participation constraint of the employee is not binding, so that the proposer chooses to offer $x_{3}=\frac{1}{4}$, while if $\frac{\delta}{3}>\frac{1}{4}$, the constraint is binding, and the employer offers $x_{3}=\frac{\delta}{3}$. Suppose now that $x_{3}=\frac{\delta}{3}$, and let us consider the case in which 2 agents are informed. As the payoff of the proposer is monotonically decreasing for shares higher than $x^{*}=\frac{1}{4}$, we have that the share offered to an employee, $x_{2}$, must satisfy

$$
K\left(x_{2}\right)^{\frac{3}{2}}\left(1-x_{2}\right)^{\frac{1}{2}}=\frac{\delta}{2} K\left(1-x_{3}\right)^{\frac{3}{2}} x_{3}^{\frac{1}{2}}
$$

If $\delta$ is not too small, we have that $x_{2} \geq x_{3}$ (for instance, if $\delta=0.9$, we have that $x_{3}=0.3$ and $x_{2}=0.311$ ). This implies that it is optimal to offer the uninformed over the other informed agent, as the equilibrium requires. Observe that both these shares improve efficiency with respect to the

\footnotetext{
${ }^{51}$ Moreover, employees always accept if and only if offered at least their continuation value. If everybody is informed, offerers always choose one employee by selecting him randomly between the other two agents with the same probability.
} 
no-leakage case as it is easy to check that the welfare objective function is increasing in $x$ up to $1 / 2$. In this example the presence of information leakage improves efficiency by guaranteeing a more equal distribution of the proceeds of an idea. 


\section{References}

[1] Aghion P. and J.Tirole (1994), "The Management of Innovation" Quarterly Journal of Economics, 109.

[2] Anton J.J.and D.A.Yao (1994), "Expropriation and Inventions: Appropriable Rents in the Absence of Property Rights" American Economics Review, 84-1.

[3] Arrow K. (1962), "Economic Welfare and the Allocation of Resources for Inventions" in "The Rate and Direction of Inventive Activity", Ed. Nelson, Princeton University Press.

[4] Areeda P. and L. Kaplov (1998), "Antitrust Analysis: Problems, Text, Cases" Boston: Little Brown.

[5] Baccara M. and R.Razin (2001), "Information Leakage and Two-Sided Investment in the Absence of Intellectual Property Rights" in progress, Mimeo, Princeton University.

[6] Baron D.P. and J.A. Ferejohn (1989), "Bargaining in Legislatures" American Political Science Review, 85 .

[7] Besen S.M. and L.J.Raskind (1991), "An Introduction to the Law and Economics of Intellectual Property" Journal of Economic Perspectives, 5-1.

[8] Boldrin M. and D.K. Levine (2002), "Perfectly Competitive Innovation", Working Paper, University of Minnesota.

[9] Chari V.V. and H.Hopenhayn (1991), "Vintage Human Capital, Growth, and the Diffusion of New Technologies", Journal of Political Economy, 99.

[10] Dasgupta P. and J. Stiglitz (1980), "Uncertainty, Industrial Structure and the Speed of R\&D" Bell Journal of Economics, 11.

[11] Fisk C. (2001), "Working Knowledge: The Employment Relationship and Corporate Ownership of Intellectual Property, 1800-1930", Mimeo, Loyola University.

[12] Gilson R.J. (2000), "The Legal Infrastructure of High Technology Industrial Silicon Valley, Route 128, and Convenantes Not to Compete" NYU Law Review, 74.

[13] Goldreich A., J. Micali. and A.Wigderson (1991), "Proofs that Yield Nothing But their Validity or All Languages in NP have Zero-Knowledge Proofs" Journal of the ACM. 
[14] Hyde A. (2000), "Working in Silicon Valley: Economic and Legal Analysis of a High-Velocity Labor Market" Unpublished Book Manuscript.

[15] Hughes J.(1988), "The Philosophy of Intellectual Property", Georgetown Law Journal, 7.

[16] Jovanovic B. and G.M. MacDonald (1994), "Competitive Diffusion", Journal of Political Economy, 102.

[17] Kumar K.B., R.G Rajan and L. Zingales (2001), "What Determines Firm Size?", Working Paper, University of Chicago.

[18] Lee T. and L. Wilde (1980), "Market Structure and Innovation: a Reformulation" Quarterly Journal of Economics, 194.

[19] Lester G. and E.Talley (2001), "Trade Secrets and Mutual Investments", Working Paper, University of Southern California.

[20] Levin R. C., A. K. Klevorick, R.R. Nelson and S.G.Winter (1984), "Survey Research on R\&D Appropriability and Technological Opprtunity: Part I", Working paper, Yale University.

[21] Loury G.C. (1979), "Market Structure and Innovation" Quarterly Journal of Economics, 93.

[22] Mehta S. R.(1998), "The Law of One Price and a Theory of the Firm: a Ricardian Perspective on Interindustry" Working Paper, Purdue University.

[23] Ordover J.A. (1991), "A Patent System for Both Diffusion and Exclusion" Journal of Economic Perspectives, 5-1.

[24] Osborne J.M. and A. Rubinstein (1990), "Bargaining and Markets" Academic Press Inc.

[25] Rajan R.G. and L.Zingales (2001), "The Firm as a Dedicated Hierarchy: A Theory of the Origin and Growth of Firms" Quarterly Journal of Economics, 116.

[26] Scotchmer S. (1991), "Standing on the Shoulders of Giants: Cumulative Research and the Patent Law" Journal of Economic Perspectives, 5-1.

[27] Scotchmer S.(1999), "Cumulative Research in Theory and Practice", Working Paper, University of California at Berkeley.

[28] Schumpeter F.(1943), "Capitalism, Socialism and Democracy" London, Unwin University Books. 
[29] Stole L.A. and J. Zweibel (1994), "Intra Firm Bargaining Under Non Binding Contracts" The Review of Economic Studies, 63.

[30] Wolinsky A. (2000), "A Theory of The Firm With Non Binding Employment Contracts" Econometrica, 69.

[31] Zabojnik J. (2000), "A Theory of Trade Secrets in Firms", Working Paper, University of Southern California. 


\section{Appendix}

\section{Definition of SSPE}

To define Symmetric Subgame Perfect Equilibria, we first have to require strategies to be anonymous. Let $\sigma_{i}$ be a mixed strategy of player $i \in N$. We say that $\sigma_{i}$ is anonymous if at any history $h_{i} \in H_{O}, \sigma_{i}\left(h_{i}\right)$ can be described by a triple $\left(n^{I}, n^{U}, \gamma\right)$, where $n^{I}$ and $n^{U}$ are the number of informed and uninformed agents getting the offer, respectively, and $\gamma$ is the vector of shares offered to each agent. ${ }^{52}$ The agents included in the offer are randomly chosen from among the two groups. ${ }^{53}$ The vector $\gamma$ has dimension $n^{I}+n^{U}$. The first $n^{I}$ elements, the shares offered to the informed agents, are all equal to $\gamma^{I}$ and the remaining $n^{U}$ elements, the shares offered to the uninformed agents, are all equal to $\gamma^{U} .^{54}$

Definition 1 A Subgame Perfect equilibrium is Symmetric if $\sigma_{i}$ is anonymous for any $i \in N$ and at any $h_{i}, h_{j} \in H_{O}$ following the same history $h \in N, \sigma_{i}\left(h_{i}\right)$ and $\sigma_{j}\left(h_{j}\right)$ can be described by the same triple $\left(n^{I}, n^{U}, \gamma\right)$. Moreover, at any $h^{\prime} \in H_{R}, \sigma_{i}\left(h^{\prime}\right)$ and $\sigma_{j}\left(h^{\prime}\right)$ are the same for any $i$ and $j$ who are playing at $h^{\prime}$.

Proof of Proposition 1: Consider the following strategy profile: every proposer always offers one uninformed agent his continuation value, and every offer is accepted if and only if an agent is offered his continuation value or more. The sequence $\left\{v_{k}\right\}_{k=2}^{n}$ of the continuation values of each informed player at the beginning of a renegotiation subgame with $k$ informed players is defined by $v_{k}=\frac{\delta}{k}\left(1-v_{k+1}\right)$ and $v_{n}=\frac{\delta}{n}$. We prove that this is the unique SSPE by backward induction on the number of people informed. Start from a subgame in which all the $n$ agents are informed. Symmetry guarantees that $v_{n}=\frac{\delta}{n}$. Let us now move to a subgame in which $n-1$ agents are informed. Consider the options of a proposer. He can form a firm with one or more informed agents, he can offer to the only uninformed agent, or he can make an offer to both informed and uninformed agents. If the offer includes the uninformed, every offered agent has to be paid $\frac{\delta}{n}$, so the uninformed is offered to. Suppose you offer to one or more informed agents instead. Upon rejection, each of them will be the next proposer with probability $\frac{1}{n-1}$, and in that case he can guarantee himself $1-\frac{\delta}{n}$ in that case. Then, an offer that does not include the uninformed agent must

\footnotetext{
${ }^{52}$ This implies that $n^{I} \in\left\{0,1, . . k\left(h_{i}\right)-1\right\}, n^{U} \in\left\{0,1, . ., n-k\left(h_{i}\right)\right\}$, and $\gamma$ is such that $\gamma \geq 0$ and $\sum_{i} \gamma_{i} \leq 1$.

${ }^{53}$ Then, since at history $h_{i}$ there are $\operatorname{card}\left(K\left(h_{i}\right) \backslash\{i\}\right)$ informed agents and $\operatorname{card}\left(N \backslash K\left(h_{i}\right)\right)$ uninformed agents, each informed agent gets the offer with probability $\frac{n^{I}}{\operatorname{card}\left(K\left(h_{i}\right) \backslash\{i\}\right)}$, and each uninformed agent gets the offer with probability $\frac{n^{U}}{\operatorname{card}\left(N \backslash K\left(h_{i}\right)\right)}$.

${ }^{54}$ More generally, we could allow for any mixture of these strategies. The results would remain the same under this alternative formulation.
} 
pay at least $\frac{\delta}{n-1}\left(1-\frac{\delta}{n}\right)$ to each agent. Since $\frac{\delta}{n-1}\left(1-\frac{\delta}{n}\right)>\frac{\delta}{n}$, offering to the uninformed dominates offering to one or more informed. This guarantees that $v_{n-1}=\frac{\delta}{n-1}\left(1-\frac{\delta}{n}\right)$. It is also easy to check that $v_{n-1}<2 v_{n}$. Let us now fix $k$ and assume as an inductive hypothesis that in all subgames starting with a number of informed agents $h \in\{k+1, . ., n-1\}$, every proposer always offers exactly one uninformed, so that we have

$$
v_{h}=\frac{\delta}{h}\left(1-v_{h+1}\right)=\frac{\delta}{h}\left(1-\frac{\delta}{h+1}(1-\ldots)\right)
$$

Let us also assume that for any $s \in\{1, . ., n-k-1\}$, we have

$$
(s+1) v_{k+s+1}>s v_{k+s}
$$

Focus on a subgame starting with $k$ informed agents. Notice that every proposer can offer to one or more uninformed agents, to one or more informed agents, or to both informed and uninformed agents. Notice that (2) guarantees that offering to one uninformed always dominates offering to more than one uninformed. Also, if an offer includes one uninformed, each agent in the offer has to be paid $v_{k+1}$. If one offers to one or more informed, each has to be paid at least $\frac{\delta}{k}\left(1-v_{k+1}\right)$. However, notice that since $v_{k+1}=\frac{\delta}{k+1}\left(1-v_{k+2}\right)$, we have $v_{k+1} \leq \frac{\delta}{k+1}$, which implies $\left(1-v_{k+1}\right) \geq\left(1-\frac{\delta}{k+1} j\right)$ and therefore

$$
\begin{aligned}
v_{k} & =\frac{\delta}{k}\left(1-v_{k+1}\right) \geq \frac{\delta}{k}\left(\frac{k+1-\delta}{k+1}\right) \\
& =\frac{\delta}{k+1}\left(\frac{k+1-\delta}{k}\right)>\frac{\delta}{k+1} \\
& \geq v_{k+1}
\end{aligned}
$$

which shows that offering to one uninformed dominates offering to one or more informed agents. Moreover, for each $s \geq 1$ we have

$$
\begin{aligned}
\frac{v_{k+s}}{v_{k+s-1}} & =\frac{\frac{\delta}{k+s}\left(1-v_{k+s+1}\right)}{\frac{\delta}{k+m-1}\left(1-v_{k+m}\right)}=\frac{k+s-1}{k+s} \frac{\left(1-v_{k+s+1}\right)}{\left(1-v_{k+s}\right)} \\
& >\frac{k+s-1}{k+s}>\frac{s}{s+1}
\end{aligned}
$$

This implies $(s+1) v_{k+s}>s v_{k+s-1}$. This concludes the proof that the candidate strategy profile is indeed the only SSPE

Proof of Corollary 2: Assume $K_{0}=\{1\}$. From Proposition 1, we know that as $n$ goes to infinity, the equilibrium sequence of continuation values $\left\{\widehat{v}_{k}\right\}_{k=2}^{\infty}$ is given by 


$$
\begin{aligned}
\widehat{v}_{k} & =\frac{\delta}{k}\left(1-\widehat{v}_{k+1}\right)=\frac{\delta}{k}\left(1-\frac{\delta}{k+1}\left(1-\frac{\delta}{k+2}\left(1-\frac{\delta}{k+3}(\ldots)\right)\right)\right) \\
& =\sum_{i=1}^{\infty} \delta^{i} \frac{(k-1) !}{(k-1+i) !}(-1)^{i-1}
\end{aligned}
$$

In particular, we have $\widehat{v}_{2}=\frac{1}{\delta}\left(e^{-\delta}-1+\delta\right)$. This implies that the payoff of the innovator is given by

$$
v(\delta) \equiv 1-v_{2}^{\infty}=1-\frac{1}{\delta}\left(e^{-\delta}-1+\delta\right)=\frac{1}{\delta}-\frac{e^{-\delta}}{\delta}>1-e^{-1}>\frac{1}{2}
$$

Notice that $v(\delta)=1$ and $\lim _{\delta \rightarrow 1} v(\delta)=.63212$

Proof of Proposition 3: The sequence $\left\{v_{k}^{m}\right\}_{k=2}^{n}$ of the continuation values of the informed players at the beginning of a renegotiation subgame with $k$ informed player is defined by $v_{n}=\frac{\delta}{n}, v_{k}^{m}=$ $\frac{\delta}{k}\left(1-(n-k) \frac{\delta}{n}\right)$ for $n-m \leq k<n$ and $v_{k}^{m}=\frac{\delta}{k}\left(1-m v_{k+m}^{m}\right)$ for $k<n-m$.

We show that this is the only SSPE by backward induction on the number of informed agents $k$. Let all the $n$ agents be informed. Then, symmetry guarantees that $v_{n}^{m}=\frac{\delta}{n}$.

Let now the number of informed people be $n-1$. Any proposer can involve the only uninformed agent in his offer or decide to offer only to informed agents. If the offers includes the uninformed, each player included in the offer has a reservation value of $\frac{\delta}{n}$.

We claim that if the offer does not involve the uninformed agent, each player has to be paid at least $\frac{\delta}{n-1}\left(1-m \frac{\delta}{n}\right)+\frac{\delta(m-1)}{n-1} \frac{\delta}{n}$. To prove this claim, observe that upon rejection, an informed agent, say $i$, will be the next proposer with probability $\frac{1}{n-1}$ and in that case he could decide to make an offer involving the uninformed, and get $\left(1-m \frac{\delta}{n}\right)$. If he is not chosen as next proposer (event that occurs with probability $\frac{n-2}{n-1}$ ), only two cases are possible: the next proposer is going to include the uninformed in the offer, or the next offer is not going to include the uninformed in the offer. In the first case, symmetry guarantees that player $i$ is going to be included in the offer with probability not inferior to $\frac{m-1}{n-2}$ (the probability is going to be greater if the offer involves more than $m$ people) and he gets $\frac{\delta}{n}$, while in the second case, symmetry guarantees that player $i$ is going to be included in the offer with probability not inferior to $\frac{m}{n-2}$ and that the least he gets is $\frac{\delta\left(1-\frac{\delta}{n}\right)}{n-1}$ as the entire pie to share among all the agents is $\delta$, while a maximum of $\frac{\delta}{n}$ can be appropriated by the uninformed. Then, we have that the expected value in the first and second case are respectively $\frac{m-1}{n-2} \frac{\delta}{n}$ and $\frac{m}{n-2} \frac{\delta\left(1-\frac{\delta}{n}\right)}{n-1}$. It is easy to see that $\frac{m-1}{n-2} \frac{\delta}{n}<\frac{m}{n-2} \frac{\delta\left(1-\frac{\delta}{n}\right)}{n-1}$. This implies our claim. In fact, if the offer does not involve the uninformed agent, each player has to be paid at least 


$$
\begin{aligned}
\frac{\delta}{n-1}\left(1-m \frac{\delta}{n}\right)+\frac{n-2}{n-1} \frac{\delta(m-1)}{n-2} \frac{\delta}{n} & =\frac{\delta}{n-1}\left(1-m \frac{\delta}{n}\right)+\frac{\delta(m-1)}{n-1} \frac{\delta}{n} \\
& =\frac{\delta}{n-1}\left(1-\frac{\delta}{n}\right) \\
& >\frac{\delta}{n}
\end{aligned}
$$

which guarantees that including the uninformed in the offer dominates not doing it. This implies that

$$
v_{n-1}^{m}=\frac{\delta}{n-1}\left(1-\frac{\delta}{n}\right)
$$

Notice that $v_{n-1}^{m}<2 v_{n}^{m}$.

Let now fix $k+1>n-m$ and assume as an inductive hypothesis that for all $n-2 \geq h \geq k+1$, we have that in all the subgames starting with $h$ agents informed, all the available $n-h$ uninformed agents are offered, and the remaining $m-n+h$ agents necessary to form the firm are chosen with equal probability among the informed ones. Also, $v_{h}^{m}>v_{h+1}^{m}$ and $s v_{h+1+s}^{m}<(s+1) v_{h+2+s}^{m}$. Let us now focus on a subgame starting with $k$ informed agents. Observe that any proposer, say $i$, can exhaust the uninformed agents and pay $\frac{\delta}{n}$ each, or decide to substitute some uninformed agents with informed ones. If he chooses the second option and he offers to, say $s>0$, uninformed agents, he has to pay each agent $v_{k+s}^{m}$. However, by our inductive hypothesis, $v_{k+h}^{m}>\frac{\delta}{n}$. This implies that offering to all uninformed dominates this option. Suppose then that $s=0$, meaning that proposer $i$ only offers to informed agents. Observe that in this case, the reservation value of each agent who gets the offer, say agent $j$, is at least $\frac{\delta}{k}\left(1-m \frac{\delta}{n}\right)+\frac{\delta(k-1)}{k} \frac{m}{k-1} \frac{\delta}{k}$. In fact, notice that symmetry guarantees that if agent $i$ does not offer to any uninformed, the other agents do not so either. This implies that $\frac{1}{k}$ is the probability of being chosen as next proposer, $1-m \frac{\delta}{n}$ is the payoff he can guarantee himself in that event, $\frac{k-1}{k}$ is the probability of not being chosen as the next proposer, $\frac{m}{k-1}$ is the probability that agent $j$ is included in the offer of someone else, and $\frac{\delta}{k}$ is the minimum value agent $j$ will be offered. However, notice that

$$
\begin{aligned}
\frac{\delta}{k}\left(1-m \frac{\delta}{n}\right)+\frac{\delta(k-1)}{k} \frac{m}{k-1} \frac{\delta}{k} & =\frac{\delta}{k}\left(1-m \frac{\delta}{n}\right)+\delta \frac{m}{k} \frac{\delta}{k} \\
& =\frac{\delta}{k}\left(1-m \frac{\delta}{n}+\delta \frac{m}{k}\right) \\
& >\frac{\delta}{n}
\end{aligned}
$$

This implies that, again, offering to all available uninformed agents dominates offering to only informed agents. For $k \geq n-m$, we have 


$$
\begin{aligned}
v_{k}^{m} & =\frac{\delta}{k}\left(1-m \frac{\delta}{n}\right)+\frac{\delta(k-1)}{k} \frac{m-n+k}{k-1} \frac{\delta}{n} \\
& =\frac{\delta}{k}\left(1-(n-k) \frac{\delta}{n}\right)
\end{aligned}
$$

Notice also that $v_{k}^{m}>v_{k+1}^{m}$ as

$$
\begin{aligned}
v_{k}^{m} & =\frac{\delta}{k}\left[1-(n-k) \frac{\delta}{n}\right] \\
& >\frac{\delta}{k+1}\left[1-(n-k-1) \frac{\delta}{n}\right] \\
& =v_{k+1}^{m}
\end{aligned}
$$

and

$$
\begin{aligned}
\frac{v_{k+1}^{m}}{v_{k}^{m}} & =\frac{k}{k+1} \frac{\left[1-(n-k-1) \frac{\delta}{n}\right]}{\left[1-(n-k) \frac{\delta}{n}\right]} \\
& >\frac{k}{k+1}
\end{aligned}
$$

which conclude the inductive proof for $k \geq n-m$.

To complete the construction of the equilibrium sequence, take as first step of the new induction the subgame in which $k=n-m$, for which we already know that

$$
v_{n-m}^{m}=\frac{\delta}{n-m}\left(1-m \frac{\delta}{n}\right)=\frac{\delta}{n-m}\left(\frac{n-\delta m}{n}\right)
$$

We have already showed that $v_{n-m}^{m}>v_{n-m+1}^{m}$ and that $\frac{v_{n-m+1}^{m}}{v_{n-m}^{m}}>\frac{n-m}{n-m+1}$. Let us now fix $k<n-m$ and assume as an inductive hypothesis that for $h \geq k+1$, we have $v_{h}^{m}>v_{h+1}^{m}$ and $\frac{v_{h+1}^{m}}{v_{h}^{m}}>\frac{h}{h+1}$, so that offers are always made to exactly $m$ uninformed agents. Focus now on a subgame starting with $k$ agents informed. If a proposer offers only to uninformed agents, he has to pay each of them $v_{k+m}^{m}$. If he offers to $s<m$ uninformed agents, he has to pay each agent $v_{k+s}^{m}$, but we know by our inductive hypothesis that $v_{k+s}^{m}>v_{k+m}^{m}$, so it is optimal to offer to all uninformed agents instead. Finally, suppose the proposer offers only to $m$ informed agents. In this case, each of them has to be paid at least $\frac{\delta}{k}\left(1-m v_{k+m}^{m}\right)$. However, notice that

$$
\frac{\delta}{k}\left(1-m v_{k+m}^{m}\right)>v_{k+m}^{m}
$$


as by inductive hypothesis $v_{k+m}^{m} \leq \frac{\delta}{k+m}$. It is then always optimal to extend the offer only to uninformed.

For $k \leq n-m$, we have

$$
v_{k}^{m}=\frac{\delta}{k}\left(1-m v_{k+m}^{m}\right)
$$

Moreover, notice that for all such $k$, we have $v_{k}^{m}<\frac{\delta}{k}$ and $v_{k}^{m}>\frac{\delta}{k}\left(\frac{k+m(1-\delta)}{k+m}\right)$. We have

$$
\begin{aligned}
\frac{v_{k-1}^{m}}{v_{k}^{m}} & =\frac{\frac{\delta}{k-1}\left(1-m v_{k-1+m}\right)}{\frac{\delta}{k}\left(1-m v_{k+m}\right)} \\
& =\frac{k}{k-1} \frac{\left(1-m v_{k-1+m}\right)}{\left(1-m v_{k+m}\right)} \\
& >\frac{k}{k-1} \frac{\left(1-m v_{k-1+m}\right)}{\left(1-m \frac{m}{m+1} v_{k-1+m}\right)}
\end{aligned}
$$

as $\frac{v_{k+m}^{m}}{v_{k+m-1}^{m}}>\frac{k+m-1}{k+m}>\frac{m}{m+1}$. Now, observe that

$$
\frac{\partial \frac{(1-m x)}{\left(1-m \frac{m}{m+1} x\right)}}{\partial x}=-m \frac{m+1}{\left(-m-1+m^{2} x\right)^{2}}<0
$$

and

$$
\frac{\partial\left[\frac{k-1+m(1-\delta)}{k-1+m\left(1-\frac{m}{m+1} \delta\right)}\right]}{\partial \delta}=\frac{-m(k-1+m)(m+1)}{\left(-(k-1) m-(k-1)-m^{2}-m+m^{2} \delta\right)^{2}}<0
$$

Therefore, we have

$$
\begin{aligned}
\frac{v_{k-1}^{m}}{v_{k}^{m}} & >\frac{k}{k-1} \frac{\left(1-m v_{k-1+m}\right)}{\left(1-m \frac{m}{m+1} v_{k-1+m}\right)} \\
& >\frac{k}{k-1} \frac{\left(1-m \frac{\delta}{k-1+m}\right)}{\left(1-m \frac{m}{m+1} \frac{\delta}{k-1+m}\right)} \\
& >\frac{k}{k-1} \frac{k-1+m(1-\delta)}{k-1+m\left(1-\frac{m}{m+1} \delta\right)} \\
& >\frac{k}{k-1} \frac{k-1}{k-1+\frac{m}{m+1}} \\
& =\frac{k}{k-1+\left(\frac{m}{m+1}\right)}>1
\end{aligned}
$$

Finally, we have $\frac{v_{k}^{m}}{v_{k-1}^{m}}>\frac{k-1}{k}$ as 


$$
\begin{aligned}
\frac{v_{k}^{m}}{v_{k-1}^{m}} & =\frac{\frac{\delta}{k}\left(1-m v_{k+m}\right)}{\frac{\delta}{k-1}\left(1-m v_{k-1+m}\right)} \\
& =\frac{k-1}{k} \frac{\left(1-m v_{k+m}\right)}{\left(1-m v_{k-1+m}\right)} \\
& >\frac{k-1}{k}
\end{aligned}
$$

Proof of Corollary 4: Focus on the case in which $n$ is large. For any number of players $n$ and any optimal firm size $m+1$, Proposition 3 guarantees that there exists a unique SSPE such that the sequence of continuation values is $\left\{v_{k}^{m}(n)\right\}_{k=1}^{n}$ Such a sequence is such that $v_{m+1}^{m}(n)$ is defined as

$$
\begin{aligned}
v_{m+1}^{m}(n) & =\frac{\delta}{m+1}\left(1-m v_{2 m+1}^{m}\right) \\
& =\frac{\delta}{m+1}\left(1-m\left(\frac{\delta}{2 m+1}\left(1-m\left(\frac{\delta}{3 m+1}(1-m(\ldots))\right)\right)\right)\right) \\
& \approx \sum_{i=1}^{\max \{j \mid j m+1 \leq n\}} \frac{\delta^{i}(m)^{i-1}(-1)^{i-1}}{\prod_{s=1}^{i}(s m+1)}
\end{aligned}
$$

This can be approximated for large $n$ by

$$
v_{m+1}^{m}(\infty)=\sum_{i=1}^{\infty} \frac{\delta^{i}(m)^{i-1}(-1)^{i-1}}{\prod_{s=1}^{i}(s m+1)}
$$

where $v_{k}^{m}(\infty) \equiv \lim _{n \rightarrow \infty} v_{k}^{m}(n)$. The innovator's appropriation rate is $v(m, \delta, \infty) \equiv 1-m v_{m+1}^{m}(\infty)$, which for large $m$ is approximated by

$$
\begin{aligned}
\lim _{m \rightarrow \infty} v(m, \delta, \infty) & =1-\lim _{m \rightarrow \infty} m \sum_{i=1}^{\infty} \frac{\delta^{i} m^{i-1}(-1)^{i-1}}{\prod_{s=1}^{i}(s m+1)}=e^{-\delta} \\
& \geq \quad e^{-1}>0
\end{aligned}
$$

As $\delta$ tends to 1 , we have $\lim _{\delta \rightarrow 1} v(\infty, \delta, \infty)=e^{-1} \simeq 0.368$

Proof of Proposition 6: Focus on a subgame where all $n$ agents are informed. Then, any proposer can offer to an uninformed agent and guarantee himself $\pi_{1}-\frac{\delta}{n}$ (by symmetry, $\frac{\delta}{n}$ is the maximum possible continuation value of another player), or he can make a grand-coalition offer. If he makes a grand coalition offer, as $\pi_{1}+\delta \pi_{2}<1$, the least he has to pay each player is $\frac{\delta}{n}\left(\pi_{1}+\delta \pi_{2}\right)$, so that the minimum cost of the offer is $(n-1) \frac{\delta}{n}\left(\pi_{1}+\delta \pi_{2}\right)$. Notice that, under our assumption, we have

$$
1-\frac{\delta(n-1)}{n}\left(\pi_{1}+\delta \pi_{2}\right) \leq \pi_{1}-\frac{\delta}{n}
$$


This implies that making a cost-minimizing offer dominates making a grand-coalition offer

Proof of Proposition 7: Suppose that $n$ agents are informed. Then, we have that making a costminimizing offer costs at least $\delta \frac{\pi_{1}+\delta \pi_{2}}{n}$ and a grand coalition offer costs at most $(n-1) \frac{\delta}{n}$. This implies that a grand coalition arises if $\pi_{1}-\delta \frac{\pi_{1}+\delta \pi_{2}}{n}<1-(n-1) \frac{\delta}{n}$, which is equivalent to $\pi_{1}\left(1-\frac{\delta}{n}\right)<$ $1-(n-1) \frac{\delta}{n}+\frac{\delta^{2} \pi_{2}}{n}=\frac{n-\delta(n-1)+\delta^{2} \pi_{2}}{n}$, or $\pi_{1}<\frac{n-(n-1) \delta+\delta^{2} \pi_{2}}{n-\delta}$, which is satisfied by assumption. Then, we have $v_{n}=\frac{\delta}{n}$. Let us move to a subgame where $n-1$ agents are informed. Any chosen proposer can offer to all the other players and pay $(n-1) \frac{\delta}{n}$, he can decide to make an offer only to the other $n-2$ informed players, paying each at most $\frac{\delta}{n-1}$ (in this case he pays at most $\left.(n-2) \frac{\delta}{n-1}<(n-1) \frac{\delta}{n}\right)$, or he can decide to offer only to the only uninformed player and get $\pi_{1}-\frac{\delta}{n}$. Observe that $\pi_{1}-\frac{\delta}{n}<1-(n-2) \frac{\delta}{n-1}$ if $\pi_{1}<1-(n-2) \frac{\delta}{n-1}+\frac{\delta}{n}$. However, we have

$$
\pi_{1}<\frac{n-(n-1) \delta+\delta^{2} \pi_{2}}{n-\delta}<1-\frac{n-2}{n} \delta<1-(n-2) \frac{\delta}{n-1}+\frac{\delta}{n}
$$

This guarantees that $v_{n-1}=\frac{\delta}{n-1}$. Assume now as an inductive hypothesis that $v_{k+1}=\frac{\delta}{k+1}$ and in all the subgames with $k+1$ or more agents informed, only informed agents receive offers. Then, focus on a subgame starting with $k$ agents informed. Every proposer has the choice of making a cost-minimizing offer, exactly a grand coalition offer or to make a more extended grand coalition offer. In the last case, he has to pay $(h-1) \frac{\delta}{h}$ with $h \geq k+1$, while with an offer made only to informed agents, he pays at most $(k-1) \frac{\delta}{k}<(h-1) \frac{\delta}{h}$ for $h \geq k+1$. Making a cost-minimizing offer yields $\pi_{1}-\frac{\delta}{k+1}$. We have that $\pi_{1}-\frac{\delta}{k+1}<1-(k-1) \frac{\delta}{k}$ if $\pi_{1}<1-(k-1) \frac{\delta}{k}+\frac{\delta}{k+1}$, but we have

$$
\pi_{1}<\frac{n-(n-1) \delta+\delta^{2} \pi_{2}}{n-\delta}<1-\frac{n-2}{n} \delta<1-(k-1) \frac{\delta}{k}+\frac{\delta}{k+1}
$$

This concludes the proof

Proof of Proposition 8 Let us build a SSPE in which at any history $h \in H_{O}$ such that $k(h)>\bar{k}$, all the informed agents at a history $h$ offer a grand coalition if chosen by nature as next offerers (subgame of type 1). If one of the players chooses to offer the grand coalition, let all the players offer an uninformed player in the subsequent subgame. To support a subgame in which all the players make an offer to an uninformed (subgame of type 2), let all the players make grand coalition offers in the subsequent subgame. Then, we have

$$
\begin{aligned}
v_{k}^{1} & =\frac{\delta}{k}\left(1-(k-1) v_{k}^{2}\right)+\frac{\delta(k-1)}{k} v_{k}^{2} \\
v_{k}^{2} & =\frac{\delta}{k}\left(\pi_{1}-v_{k+1}^{1}\right)+\frac{\delta(k-1)}{k} \pi_{2} v_{k-1}^{*}
\end{aligned}
$$


Notice that $\lim _{k} v_{k}^{1}=\lim _{k} v_{k}^{2}=0$. To check if a subgame of type 1 is a SSPE we show that

$$
1-(k-1) v_{k}^{2}>\pi_{1}
$$

Notice that $\lim _{k}\left[1-(k-1) v_{k}^{2}\right]=1-\delta \pi_{1}-\delta^{2} \pi_{2}>\pi_{1}$, so for high $k$ (3) is satisfied. Also, we need to check that it is not optimal to offer to more than the grand coalition, but we can sustain this by imposing a type 1 subgame upon rejection. Therefore we must have

$$
1-(k-1) v_{k}^{2}>1-(k+m-1) v_{k+m}^{1}
$$

or

$$
(k-1) v_{k}^{2}<(k+m-1) v_{k+m}^{1}
$$

This is satisfied as $\lim _{k}(k-1) v_{k}^{2}=\delta \pi_{1}+\delta^{2} \pi_{2}$ and $\lim _{k \rightarrow \infty}(k+m-1) v_{k+m}^{1}=\delta$. Moreover, we know that $\delta \pi_{1}+\delta^{2} \pi_{2}>\delta$ as $\pi_{1}<\frac{1-\delta^{2} \pi_{2}}{1+\delta}<1-\delta \pi_{2}$.

Now, to check the sustainability of the subgames of type 2 , we need to check that

$$
\pi_{1}-v_{k+1}^{1}>1-(k-1) v_{k}^{1}
$$

But we have $\lim _{k}\left[1-(k-1) v_{k}^{1}\right]=\lim _{k \rightarrow \infty}\left\{1-(k-1)\left[\frac{\delta}{k}\left(1-(k-1) v_{k}^{2}\right)+\frac{\delta(k-1)}{k} v_{k}^{2}\right]\right\}$ $=1-\delta<\pi_{1}$, so that (4) is satisfied as well.

Proof of Proposition 9:We need to show that there is $\tilde{\pi}\left(\pi_{2}, \delta, n\right)<1$ such that if $\pi_{1}>\tilde{\pi}\left(\pi_{2}, \delta, n\right)$, then always offering one uninformed agent is an equilibrium. In this strategy profile, the continuation value sequence is $\left\{v_{k}\right\}_{k=2}^{n}$ defined by $v_{n}=\frac{\delta}{n}\left(\pi_{1}+\delta \pi_{2}\right)$ and $v_{k}=\frac{\delta}{k}\left(\pi_{1}-v_{k+1}\right)+\frac{\delta(k-1)}{k} \pi_{2} v_{k-1}^{\star}$, where we denote by $\left\{v_{k}^{*}\right\}_{k=2}^{n}$ the (unique) SSPE sequence we studied in the $\pi_{1}=1, \pi_{2}=0$ case (observe that after the formation of the first firm, if $k$ informed agents are left on the market, each agent has a continuation value of $\delta \pi_{2} v_{k}^{*}$.) To show that this is an equilibrium, we need to prove that the sequence $\left\{v_{k}\right\}_{k=2}^{n}$ is decreasing, that $m v_{k+m}<(m+1) v_{k+m+1}$ for all $k$ and $m$ such that $1 \leq$ $m \leq n-k-1$, and that offering to one uninformed always dominates making a grand-coalition offer. Let us first show that the sequence $\left\{v_{k}\right\}_{k=2}^{n}$ is decreasing and that $m v_{k+m}<(m+1) v_{k+m+1}$ for all $k, m$. To see that $\left\{v_{k}\right\}_{k=2}^{n}$ is decreasing, notice that we have $v_{k}=\frac{\delta}{k}\left(\pi_{1}-v_{k+1}\right)+\frac{\delta(k-1)}{k} \pi_{2} v_{k-2}^{*}$ and $v_{n}=\frac{\delta}{n}\left(\pi_{1}-v_{n}\right)+\frac{\delta(n-1)}{n}\left(\frac{1}{n-1} v_{n}+\frac{n-2}{n-1} \pi_{2} v_{n-2}^{*}\right)$ where $v_{n-2}^{*}=\frac{\delta}{n-2}$. Therefore, $v_{n}=\frac{\delta}{n}\left(\pi_{1}+\delta \pi_{2}\right)$ and for any $k \leq n$ we have 


$$
\begin{aligned}
\lim _{\pi_{1} \rightarrow 1} v_{k} & =\lim _{\pi_{1} \rightarrow 1} \frac{\delta}{k}\left(\pi_{1}-v_{k+1}\right)+\frac{\delta(k-1)}{k} \pi_{2} v_{k-2}^{*}= \\
& =v_{k}^{*}>v_{k+1}^{*}=\lim _{\pi_{1} \rightarrow 1} v_{k+1}
\end{aligned}
$$

In the same way, it is easy to see that

$$
\lim _{\pi_{1} \rightarrow 1}\left[(m+1) v_{k+m+1}\right]>\lim _{\pi_{1} \rightarrow 1}\left[v_{k+1} m v_{k+m}\right]
$$

for all $1 \leq m \leq n-k-1$. So, there exists $\pi^{\prime}\left(\pi_{2}, \delta, n\right)<1$ such that for all $\pi_{1} \geq \pi^{\prime}\left(\pi_{2}, \delta, n\right)$ the sequence $\left\{v_{k}\right\}_{k=2}^{n}$ is decreasing and satisfies $m v_{k+m}<(m+1) v_{k+m+1}$.

To show that offering to one uninformed always dominates making a grand-coalition offer we need

$$
\pi_{1}-\frac{\delta\left(\pi_{1}-v_{k+2}\right)+\delta k \pi_{2} v_{k}^{*}}{k+1}>1-(k-1) \frac{\delta\left(\pi_{1}-v_{k+1}\right)+\delta(k-1) \pi_{2} v_{k-1}^{*}}{k}
$$

for all $k$. Notice that

$$
\begin{aligned}
\lim _{\pi_{1} \rightarrow 1}\left[\pi_{1}-\frac{\delta\left(\pi_{1}-v_{k+2}\right)+\delta k \pi_{2} v_{k}^{*}}{k+1}\right] & 1-v_{k+1}^{*} \\
> & 1-(k-1) v_{k}^{*}= \\
& \lim _{\pi_{1} \rightarrow 1}\left[1-(k-1) \frac{\delta\left(\pi_{1}-v_{k+1}\right)+\delta(k-1) \pi_{2} v_{k-1}^{*}}{k}\right]
\end{aligned}
$$

for all $k$. So, by continuity with respect to $\pi_{1}$, there is $\pi^{\prime \prime}\left(\pi_{2}, \delta, n\right)<1$, such that for all $\pi_{1} \geq$ $\pi^{\prime \prime}\left(\pi_{2}, \delta, n\right),(5)$ is satisfied for all $k$. Define the bound $\widetilde{\pi}\left(\pi_{2}, \delta, n\right) \equiv \max \left[\pi^{\prime}\left(\pi_{2}, \delta, n\right), \pi^{\prime \prime}\left(\pi_{2}, \delta, n\right)\right]$.

Notice that the generic element of the sequence $\left\{v_{k}\right\}_{k=2}^{n}$ for $n$ going to infinity is

$$
\begin{aligned}
v_{k}= & \frac{\delta}{k}\left(\pi_{1}-v_{k+1}\right)+\frac{\delta(k-1)}{k} \pi_{2} v_{k-1}^{\star} \\
= & \frac{\delta}{k}\left[\pi_{1}-\left(\frac{\delta}{k+1}\left(\pi_{1}-\ldots\right)+\frac{\delta k}{k+1} \pi_{2} v_{k}^{\star}\right)\right]+\frac{\delta(k-1)}{k} \pi_{2} v_{k-1}^{\star} \\
= & \sum_{i=1}^{\infty}\left[\frac{\pi_{1}(-1)^{i-1} \delta^{i}(k-1) !}{(k-1+i) !}\right. \\
& \left.\left.+\frac{\delta^{i}}{k}\left(1+(k+i) \pi_{2} \sum_{j=1}^{\infty} \delta^{j} \frac{(k+i) !}{(k+i+j) !}(-1)^{j-1}\right)\right)\right]
\end{aligned}
$$

which is increasing in both $\pi_{1}$ and $\pi_{2}$. This implies that the appropriation rate of the innovator, $v(\delta)=1-v_{2}$, is decreasing in $\pi_{1}$

Proof of Proposition 11: Let $\pi_{1} \in\left[\underline{\pi}\left(\pi_{2}, \delta, n\right), \bar{\pi}\left(\pi_{2}, \delta, n\right)\right]$, and consider the strategy profile in which as long as at least two agents are informed, everybody always offers to all the other agents. If 
someone does not, when $2<k \leq n-1$, then upon rejection we go in grand coalition stage (which by Proposition ??, we know it is sustainable either with another grand coalition stage upon rejection, or with alternating stages). As soon as along the equilibrium path everybody is informed, if a proposer offers to one agent, upon rejection all agents offer to one agents (competition arises). If a grand coalition is offered instead, we have a grand coalition arising upon rejection. To show that this is an equilibrium, start from a subgame where everybody is informed. Notice that since $\underline{\pi}\left(\pi_{2}, \delta, n\right)<\pi_{1}<\bar{\pi}\left(\pi_{2}, \delta, n\right)$, we can sustain both competition and grand coalition in that stage. To sustain competition, let competition arise if an uninformed is offered, and let grand coalition arise if a grand coalition offer is made. Then, one prefers to offer to just one agent since

$$
\pi_{1}-\frac{\delta}{n}\left(\pi_{1}+\delta \pi_{2}\right)>1-(n-1) \frac{\delta}{n}
$$

is satisfied as $\pi_{1}>\underline{\pi}\left(\pi_{2}, \delta, n\right)$. To sustain a grand coalition offer, let competition arise upon rejection, while if another offer is made, let a grand coalition arise. Then, we need

$$
\pi_{1}-\frac{\delta}{n}<1-\frac{(n-1)}{n} \delta\left(\pi_{1}+\delta \pi_{2}\right)
$$

which is satisfied as $\pi_{1}<\bar{\pi}\left(\pi_{2}, \delta, n\right)$.

Also, notice that if $k$ people are informed, since proposers offer to everybody else, we have

$$
\begin{aligned}
v_{k} & =\frac{\delta}{k}\left[1-(n-1) \frac{\delta}{n}\left(\pi_{1}+\delta \pi_{2}\right)\right]+\frac{\delta(k-1)}{k} \frac{\delta}{n}\left(\pi_{1}+\delta \pi_{2}\right)= \\
& =\frac{\delta}{k}-\frac{\delta^{2}}{n k}(n-k)\left(\pi_{1}+\delta \pi_{2}\right)
\end{aligned}
$$

To sustain this behavior, since when one offers to uninformed agents, we go into a grand coalition stage, we need

$$
\pi_{1}-\frac{\delta}{k+1}<1-(n-1) \frac{\delta}{n}\left(\pi_{1}+\delta \pi_{2}\right)
$$

which is guaranteed to be true by $\pi_{1}<\bar{\pi}\left(\pi_{2}, \delta, n\right)$..

Now, if the one described is the continuation of the game, the innovator can offer to one other agent. If he does so, he gets $1-v_{2}$ where

$$
\begin{aligned}
v_{2} & =\frac{\delta}{2}\left[1-(n-1) \frac{\delta}{n}\left(\pi_{1}+\delta \pi_{2}\right)\right]+\frac{\delta}{2} \frac{\delta}{n}\left(\pi_{1}+\delta \pi_{2}\right)= \\
& =\frac{\delta}{2}-(n-2) \frac{\delta^{2}}{2 n}\left(\pi_{1}+\delta \pi_{2}\right)<\frac{\delta}{2}
\end{aligned}
$$


In this equilibrium the innovator gets at least

$$
1-\frac{\delta}{2}+(n-2) \frac{\delta^{2}}{2 n}\left(\pi_{1}+\delta \pi_{2}\right)>1-\frac{\delta}{2}
$$

which is increasing in $\pi_{1}$ and $\pi_{2}$. By having competition sustainable in the last step, we have built an equilibrium in which appropriation rate of the innovator is higher than $1-\frac{\delta}{2}$. For high $n$, the innovator can do even better than that. In fact, if we let $\widetilde{k}=\min [2, \underline{k}]$, where $\underline{k}$ is the smallest $k \leq n-1$, such that $\pi_{1} \geq 1-(k-1) \frac{\delta}{k}+\frac{\delta}{k+1}$, we can sustain competition arising when there are $\widetilde{k}$ agents informed, so that the innovator can also offer $\widetilde{k}-1$ agents $\frac{\delta}{k}\left(\pi_{1}+\delta \pi_{2}\right)$ and get $1-(\widetilde{k}-1) v_{\widetilde{k}} \geq 1-(\widetilde{k}-1) \frac{\delta}{k}\left(\pi_{1}+\delta \pi_{2}\right)$, which is decreasing in $\pi_{1}$ and $\pi_{2}$. Notice that

$$
\begin{aligned}
1-(\widetilde{k}-1) \frac{\delta}{\widetilde{k}}\left(\pi_{1}+\delta \pi_{2}\right) & >1-(n-1) \frac{\delta}{n}\left(\pi_{1}+\delta \pi_{2}\right) \\
& >1-\frac{\delta}{2}+(n-2) \frac{\delta^{2}}{2 n}\left(\pi_{1}+\delta \pi_{2}\right)
\end{aligned}
$$

if $\left(\pi_{1}+\delta \pi_{2}\right)<\frac{n}{n(2+\delta)-2(1+\delta)}$ which is always satisfied for high $n$ since $\pi_{1}+\delta \pi_{2}<1$.

Let now show that if $\pi_{1}<\pi\left(\pi_{2}, \delta, n\right)=\frac{n+\delta-(n-1) \delta^{2} \pi_{2}}{n+(n-1) \delta}$, there is always an equilibrium in which the innovator appropriates $1-\frac{\delta}{2}$. First, let $\pi_{1}<1-\frac{n-2}{n} \delta$, and conjecture an equilibrium in which the grand coalition is always offered. If everybody is informed, observe that in our hypothesis, $\pi_{1}-\frac{\delta}{n}<1-(n-1) \frac{\delta}{n}$. This implies that offering the grand coalition is sustainable. Then, we can work backward by induction and, using the fact that $1-\frac{n-2}{n} \delta<1-(k-1) \frac{\delta}{k}+\frac{\delta}{k+1}$ for all $k \leq n-1$, we can show that offering always exactly to the grand coalition is sustainable again. Then, we have that the innovator appropriates $1-\frac{\delta}{2}$

Now, assume that $1-\frac{n-2}{n} \delta<\pi_{1}<\bar{\pi}\left(\pi_{2}, \delta, n\right)$. First, let $\widetilde{k}=\min [2, \underline{k}]$ where $\underline{k}$ is the smallest $k \leq n-1$, such that $\pi_{1} \geq 1-(k-1) \frac{\delta}{k}+\frac{\delta}{k+1}$. Then, every time $k \geq \widetilde{k}$ and we enter the first subgame in which $k$ people are informed, let every proposer offer to the grand coalition. Upon rejection, let every proposer offer to one uninformed agent and so on. If $k<\widetilde{k}$, let the agents always make a grand coalition offer. To check that this is an equilibrium, let us first check any $k \geq \widetilde{k}$. We have that

$$
1-(k-1) v_{k} \geq 1-(k-1) \frac{\delta\left(\pi_{1}+\delta \pi_{2}\right)}{k} \geq \pi_{1}-\frac{\delta}{k+1}
$$

which is equivalent to $\pi_{1}<\frac{1-\frac{k-1}{k} \delta^{2} \pi_{2}+\frac{\delta}{k+1}}{1+\frac{k-1}{k} \delta}$, which is satisfied as $\frac{1-\frac{k-1}{k} \delta^{2} \pi_{2}+\frac{\delta}{k+1}}{1+\frac{k-1}{k} \delta}>\frac{n+\delta-(n-1) \delta^{2} \pi_{2}}{n+(n-1) \delta}$. Upon rejection, we have that proposers offer to one uninformed as

$$
\pi_{1}-\frac{\delta}{k+1} \geq 1-(k-1) \frac{\delta}{k}
$$


which is equivalent to $\pi_{1} \geq 1-(k-1) \frac{\delta}{k}+\frac{\delta}{k+1}$, which is satisfied by definition of $\widetilde{k}$. If $k<\widetilde{k}$, we have

$$
\pi_{1}-\frac{\delta}{k+1}<1-(k-1) \frac{\delta}{k}
$$

so that a grand coalition offer dominates offering to an uninformed. Notice also that as $(k-1) \frac{\delta\left(\pi_{1}+\delta \pi_{2}\right)}{k}<$ $(k-1) \frac{\delta}{k}<k \frac{\delta}{k+1}$, it is never optimal to offer to more than the grand coalition and, as $\frac{\delta}{k+1}<m \frac{\delta}{k+m}$, it is never optimal to offer to more than one uninformed. In this equilibrium, the innovator's payoff is again $1-\frac{\delta}{2}$ 